\title{
The hadronic vacuum polarization contribution to the muon $g-2$ from lattice QCD
}

\author{
M. Della Morte, ${ }^{a}$ A. Francis, ${ }^{b}$ V. Gülpers, ${ }^{c}$ G. Herdoíza, ${ }^{d}$ G. von Hippel, ${ }^{e}$ H. Horch, ${ }^{d}$ \\ B. Jäger, ${ }^{f}$ H.B. Meyer, ${ }^{e, g}$ A. Nyffeler ${ }^{e}$ and H. Wittig ${ }^{e, g}$ \\ ${ }^{a}$ CP3-Origins, University of Southern Denmark, \\ Campusvej 55, 5230 Odense M, Denmark \\ ${ }^{b}$ Department of Physics and Astronomy, York University, \\ Toronto, ON, M3J1P3, Canada \\ ${ }^{c}$ School of Physics and Astronomy, University of Southampton, \\ Southampton SO17 1BJ, U.K. \\ ${ }^{d}$ Instituto de Física Teórica UAM/CSIC and Departamento de Física Teórica, \\ Universidad Autónoma de Madrid, \\ Cantoblanco, E-28049 Madrid, Spain \\ ${ }^{e}$ PRISMA Cluster of Excellence and Institut für Kernphysik, \\ Johann Joachim Becher-Weg 45, University of Mainz, D-55099 Mainz, Germany \\ ${ }^{f}$ ETH Zürich, Institute for Theoretical Physics, \\ Wolfgang-Pauli-Str. 27, 8093 Zürich, Switzerland \\ ${ }^{g}$ Helmholtz Institute Mainz, University of Mainz, \\ D-55099 Mainz, Germany \\ E-mail: dellamor@cp3-origins.net, afranc@yorku.ca, \\ V.M.Guelpers@soton.ac.uk, gregorio.herdoiza@uam.es, \\ hippel@uni-mainz.de, horchh@uni-mainz.de, bejaeger@itp.phys.ethz.ch, \\ meyerh@uni-mainz.de, nyffeler@uni-mainz.de, \\ hartmut.wittig@uni-mainz.de
}

ABSTRACT: We present a calculation of the hadronic vacuum polarization contribution to the muon anomalous magnetic moment, $a_{\mu}^{\text {hvp }}$, in lattice QCD employing dynamical up and down quarks. We focus on controlling the infrared regime of the vacuum polarization function. To this end we employ several complementary approaches, including Padé fits, time moments and the time-momentum representation. We correct our results for finitevolume effects by combining the Gounaris-Sakurai parameterization of the timelike pion form factor with the Lüscher formalism. On a subset of our ensembles we have derived an upper bound on the magnitude of quark-disconnected diagrams and found that they decrease the estimate for $a_{\mu}^{\text {hvp }}$ by at most $2 \%$. Our final result is $a_{\mu}^{\text {hvp }}=\left(654 \pm 32_{-23}^{+21}\right)$. 
$10^{-10}$, where the first error is statistical, and the second denotes the combined systematic uncertainty. Based on our findings we discuss the prospects for determining $a_{\mu}^{\text {hvp }}$ with sub-percent precision.

KEYwORDS: Lattice QCD, Nonperturbative Effects, Precision QED

ARXIV EPRINT: 1705.01775 


\section{Contents}

1 Introduction 1

2 Lattice approaches to $a_{\mu}^{\text {hvp }} \quad 3$

3 Simulation details 5

4 Calculation of $a_{\mu}^{\text {hvp }} \quad 8$

$4.1 a_{\mu}^{\text {hvp }}$ from the hybrid method 9

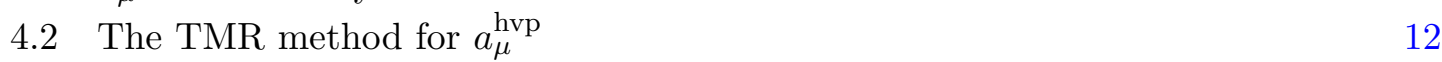

4.3 Gounaris-Sakurai based extension of the vector correlator 16

$\begin{array}{ll}4.4 \text { Comparison of } a_{\mu}^{\text {hvp }} & 17\end{array}$

5 Chiral and continuum extrapolations 18

6 Conclusions 23

A Renormalization of the vector current $\quad 24$

B The QED kernel in the time-momentum representation $\quad 24$

$\begin{array}{ll}\text { B.1 Derivation of a representation of the kernel function } & 25\end{array}$

B.2 Sensitivity of $a_{\mu}^{\text {hvp }}$ to the lattice scale setting 27

C Finite-size effects in the time-momentum representation 28

$\begin{array}{lll}\text { C.1 Finite-volume corrections for non-interacting pions } & 29\end{array}$

$\begin{array}{lll}\text { C.2 Reliability of the estimate of finite-size effects } & 31\end{array}$

C.3 Single-exponential extension of the time-momentum correlator 31

C.4 Uncertainty in the determination of the $\rho$-mass and decay width 32

D Determination of the quark-disconnected contribution 32

\section{Introduction}

After the discovery of the Higgs boson the search for physics beyond the Standard Model has further intensified. The three principal strategies include the observation of new particles, the detection of enhanced signals in rare decay processes and deviations between experimental determinations of precision observables and theoretical predictions based on the Standard Model. One of the most prominent examples for the latter is the value of the anomalous magnetic moment of the muon, $a_{\mu}=\frac{1}{2}(g-2)_{\mu}$, which exhibits a persistent deviation of $3.6 \sigma$ at the current precision of $0.5 \mathrm{ppm}$ [1]. It is well known that the theoretical uncertainty is dominated by hadronic contributions, more specifically the hadronic vacuum polarization and hadronic light-by-light scattering contributions, $a_{\mu}^{\text {hvp }}$ and $a_{\mu}^{\text {hlbl }}$, 
respectively. The estimate for $a_{\mu}^{\text {hvp }}$ which enters the Standard Model prediction is typically obtained from dispersion theory using the experimentally determined cross section $e^{+} e^{-} \rightarrow$ hadrons as input [2-7]. Recently it was proposed to extract the photon vacuum polarization in the spacelike region from Bhabha and $\mu e$ scattering data $[8,9]$, which would allow for a direct comparison with lattice results. Other approaches that combine phenomenological constraints with information from lattice QCD employ expansions of $a_{\mu}^{\text {hvp }}$ in terms of Mellin-Barnes moments [10-12] or finite energy sum rules [13, 14]. The hadronic light-by-light scattering contribution has so far only been determined via model estimates (as reviewed in [5, 15-17]), although efforts have been undertaken to move towards a data-driven [18-27] approach as well.

The determination of the hadronic contributions to the muon $(g-2)$ from first principles using lattice QCD has been the focus of many recent studies. This concerns both $a_{\mu}^{\text {hvp }}$, studied in [28-40], as well as $a_{\mu}^{\text {hlbl }}$ [41-49]. Lattice calculations of $a_{\mu}^{\text {hvp }}$ proceed by evaluating a convolution integral over Euclidean momenta $Q^{2}[28,50]$. The integral receives its dominant contribution from the region where $Q^{2} \approx m_{\mu}^{2}$, which is far below the smallest Fourier momenta that can be realized on typical lattice sizes. Therefore, lattice calculations of $a_{\mu}^{\text {hvp }}$ suffer from the additional difficulty of controlling the small-momentum regime. Various strategies for a model-independent description of the small- $Q^{2}$ regime have been discussed in the literature [32, 34, 51-56].

In this paper we present results for $a_{\mu}^{\text {hvp }}$ in lattice QCD, using two complementary approaches: the first is based on the standard determination of the vacuum polarization function $\Pi\left(Q^{2}\right)$ via a four-dimensional Fourier transform of the vector correlator. The second method uses the so-called "time-momentum representation" (TMR) discussed in $[51,54,57]$. As another variant we consider time moments of the vector correlator [34] to describe the low-momentum region of $\Pi\left(Q^{2}\right)$. We focus primarily on controlling the various sources of systematic uncertainties associated with the lattice approach to $a_{\mu}^{\text {hvp }}$, and in particular the problem of constraining the deep infrared region.

Our work is based on QCD with two light degenerate dynamical quarks. The inclusion of the effects from isospin breaking and from dynamical $s, c$ and $b$ quarks is left for future work. Clearly, for a precision determination of $a_{\mu}^{\text {hvp }}$ in lattice QCD it is necessary to include dynamical strange and charm quarks. However, the collection of results for a wide range of quantities in [58] suggests that the effects from the strange and charm quarks in the sea can be expected to be subleading at our level of precision. While the calculation of quarkdisconnected diagrams has only been performed on a subset of our ensembles, this has still allowed us to derive an upper bound on their overall influence which is included in the final error estimate. Our main result, stated in eq. (5.5), is the determination of $a_{\mu}^{\text {hvp }}$ with an overall precision of $6 \%$. While this is still significantly larger than the quoted uncertainty of the dispersive approach, our study provides valuable insights for future lattice calculations of this important quantity.

This paper is organized as follows: in section 2 we discuss different approaches for computing the hadronic vacuum polarization contribution to $(g-2)_{\mu}$. Simulation details are described in section 3, and in section 4 we present a detailed discussion and comparison of our results obtained on individual ensembles. The extrapolation of our results to 
the physical point is described in section 5, including a detailed discussion of systematic errors. We state our conclusions in section 6 . In a series of appendices we present further details on the current renormalization, the efficient evaluation of the QED kernel in the TMR, the estimation of finite-volume effects and the calculation of quark-disconnected diagrams, respectively.

\section{$2 \quad$ Lattice approaches to $a_{\mu}^{\text {hvp }}$}

The hadronic vacuum polarization contribution, $a_{\mu}^{\text {hvp }}$, to the muon anomalous magnetic moment can be obtained from the vacuum polarization function $\Pi\left(Q^{2}\right)$ convoluted with a known kernel function $K\left(Q^{2} ; m_{\mu}^{2}\right)$ (defined in appendix B) and integrated over Euclidean momenta $Q^{2}[28,50,59]$, as

$$
a_{\mu}^{\mathrm{hvp}}=4 \alpha^{2} \int_{0}^{\infty} d Q^{2} K\left(Q^{2} ; m_{\mu}^{2}\right)\left\{\Pi\left(Q^{2}\right)-\Pi(0)\right\},
$$

where $\alpha$ and $m_{\mu}$ are the electromagnetic coupling and muon mass, respectively. The vacuum polarization function $\Pi\left(Q^{2}\right)$ is obtained from the vacuum polarization tensor $\Pi_{\mu \nu}(Q)$, which is given in terms of the correlator of the electromagnetic current $J_{\mu}(x)$ as

$$
\begin{aligned}
\Pi_{\mu \nu}(Q) & =\int d^{4} x \mathrm{e}^{i Q \cdot x}\left\langle J_{\mu}(x) J_{\nu}(0)\right\rangle, \\
J_{\mu}(x) & =\frac{2}{3} \bar{u}(x) \gamma_{\mu} u(x)-\frac{1}{3} \bar{d}(x) \gamma_{\mu} d(x)-\frac{1}{3} \bar{s}(x) \gamma_{\mu} s(x)+\ldots,
\end{aligned}
$$

where $Q$ denotes the Euclidean momentum. Euclidean $\mathrm{O}(4)$ invariance and current conservation imply

$$
\Pi_{\mu \nu}(Q)=\left(Q_{\mu} Q_{\nu}-\delta_{\mu \nu} Q^{2}\right) \Pi\left(Q^{2}\right) .
$$

The subtracted vacuum polarization $\hat{\Pi}\left(Q^{2}\right)$, defined by

$$
\hat{\Pi}\left(Q^{2}\right) \equiv 4 \pi^{2}\left(\Pi\left(Q^{2}\right)-\Pi(0)\right)
$$

which appears in the integrand, is free of UV divergences. Using the explicit expression for the kernel function [28,60] one infers that the integrand in eq. (2.1) is peaked near $Q^{2} \approx m_{\mu}^{2} \approx 0.01 \mathrm{GeV}^{2}$. To access such small momenta on a finite lattice directly would require volumes corresponding to a linear extent of $\mathrm{O}(10 \mathrm{fm})$ or more, which is difficult to achieve with currently available resources. Therefore, the exact shape of $\Pi\left(Q^{2}\right)$ in the small-momentum region, as well as the value of $\Pi(0)$ are difficult to determine with sufficient accuracy.

Several methods for accurately constraining the small-momentum regime have been proposed and studied. This includes the use of twisted boundary conditions [61-63] that are designed to penetrate more deeply into the region near $Q^{2}=0[32,64,65]$, and the direct determination of the additive renormalization $\Pi(0)$, either via operator insertions [53] or by computing time moments of the vector correlator [34]. In order to avoid introducing any model dependence it has been proposed to represent $\Pi\left(Q^{2}\right)$ by either Padé approximants 
or conformal polynomials in a sub-interval $0 \leq Q^{2} \leq Q_{\text {cut }}^{2}$ and to evaluate the convolution integral for momenta $Q^{2}>Q_{\text {cut }}^{2}$ using the trapezoidal rule [56]. Such a "hybrid strategy" requires accurate data for sufficiently small values of $Q_{\text {cut }}^{2}$.

In the so-called "time-momentum representation" (TMR) discussed in $[51,54,57]$ the subtracted vacuum polarization function $\hat{\Pi}\left(Q^{2}\right)$ is directly obtained from the spatially summed two-point correlator $G\left(x_{0}\right)$ of the electromagnetic current, i.e.

$$
\begin{aligned}
& \hat{\Pi}\left(Q^{2}\right)=4 \pi^{2} \int_{0}^{\infty} d x_{0} G\left(x_{0}\right)\left[x_{0}^{2}-\frac{4}{Q^{2}} \sin ^{2}\left(\frac{1}{2} Q x_{0}\right)\right], \\
& G\left(x_{0}\right) \delta_{k l}=-\int d^{3} x\left\langle J_{k}(x) J_{l}(0)\right\rangle .
\end{aligned}
$$

When inserted into eq. (2.1), the hadronic vacuum polarization $a_{\mu}^{\text {hvp }}$ is given by

$$
a_{\mu}^{\mathrm{hvp}}=\left(\frac{\alpha}{\pi}\right)^{2} \int_{0}^{\infty} d x_{0} G\left(x_{0}\right) \widetilde{K}\left(x_{0} ; m_{\mu}\right),
$$

where the $x_{0}$-dependent kernel function $\widetilde{K}\left(x_{0} ; m_{\mu}\right)$ is obtained by performing the integral

$$
\widetilde{K}\left(x_{0} ; m_{\mu}\right)=4 \pi^{2} \int_{0}^{\infty} d Q^{2} K\left(Q^{2} ; m_{\mu}^{2}\right)\left[x_{0}^{2}-\frac{4}{Q^{2}} \sin ^{2}\left(\frac{Q x_{0}}{2}\right)\right],
$$

and $K\left(Q^{2} ; m_{\mu}^{2}\right)$ is the same kernel function as in eq. (2.1). A representation of $\widetilde{K}\left(x_{0} ; m_{\mu}\right)$ suitable for a numerical evaluation is given in appendix B. The main technical difficulty in this approach arises from the fact that the vector correlator $G\left(x_{0}\right)$ is integrated to infinite Euclidean time. Therefore, the large- $x_{0}$ behaviour of $G\left(x_{0}\right)$ must be accurately constrained. For light enough pion masses the vector correlator is dominated by the two-pion state as $x_{0} \rightarrow \infty$, and thus one has to resort to elaborate calculations of $G\left(x_{0}\right)$ including multiparticle states [51].

A closely related method for determining the subtracted vacuum polarization function $\hat{\Pi}\left(Q^{2}\right)$ is based on the calculation of the time moments of the vector correlator [34]. The starting point is the expansion of $\Pi\left(Q^{2}\right)$ at low $Q^{2}$, i.e.

$$
\Pi\left(Q^{2}\right)=\Pi_{0}+\sum_{j=1}^{\infty} \Pi_{j} Q^{2 j} .
$$

When $Q$ is chosen as $Q=(\omega, \overrightarrow{0})$ one obtains the vacuum polarization function (VPF) from the spatially summed vector correlator $G\left(x_{0}\right)$ according to

$$
\omega^{2} \Pi\left(\omega^{2}\right)=\int_{-\infty}^{\infty} d x_{0} \mathrm{e}^{i \omega x_{0}} G\left(x_{0}\right)
$$

The expansion coefficients $\Pi_{0}, \Pi_{1}, \Pi_{2}, \ldots$ in eq. (2.9) can be determined from the derivatives with respect to $\omega^{2}$ which are, in turn, related to the time moments $G_{2 j}$ of the vector correlator via

$$
G_{2 j}:=\int_{-\infty}^{\infty} d x_{0} x_{0}^{2 j} G\left(x_{0}\right)=(-1)^{j} \frac{\partial^{2 j}}{\partial \omega^{2 j}}\left\{\omega^{2} \Pi\left(\omega^{2}\right)\right\}_{\omega^{2}=0} .
$$


In this way one obtains

$$
\Pi(0) \equiv \Pi_{0}=-\frac{1}{2} G_{2}, \quad \Pi_{j}=(-1)^{j+1} \frac{G_{2 j+2}}{(2 j+2) !}, \quad j=1,2, \ldots
$$

The time moments can be used to construct the Padé representation of the subtracted VPF $\hat{\Pi}\left(Q^{2}\right) \equiv 4 \pi^{2}\left(\Pi\left(Q^{2}\right)-\Pi(0)\right)$ in the low-momentum regime. There is also a close relation between time moments and the TMR: by expanding the sine function in eq. (2.5) as a power series in $Q^{2}$ one recovers the time moments as expansion coefficients in accordance with eq. (2.9).

For later use it is also convenient to consider the decomposition of the electromagnetic current into an iso-vector $(I=1)$ and an iso-scalar $(I=0)$ part, according to

$$
\begin{aligned}
J_{\mu}(x) & =J_{\mu}^{\rho}(x)+J_{\mu}^{I=0}(x), \\
J_{\mu}^{\rho}(x) & =\frac{1}{2}\left(\bar{u} \gamma_{\mu} u-\bar{d} \gamma_{\mu} d\right), \quad J_{\mu}^{I=0}(x)=\frac{1}{6}\left(\bar{u} \gamma_{\mu} u+\bar{d} \gamma_{\mu} d-2 \bar{s} \gamma_{\mu} s+\ldots\right),
\end{aligned}
$$

where we use the superscript $\rho$ to denote the iso-vector $(I=1)$ contribution. The corresponding correlator is defined by

$$
G^{\rho \rho}\left(x_{0}\right) \delta_{k l}=-\int d^{3} x\left\langle J_{k}^{\rho}(x) J_{l}^{\rho}(0)\right\rangle
$$

and the iso-spin decomposition of the vector correlator reads

$$
G\left(x_{0}\right)=G^{\rho \rho}\left(x_{0}\right)+G^{I=0}\left(x_{0}\right) .
$$

Note that only quark-connected diagrams contribute to the iso-vector correlator $G^{\rho \rho}\left(x_{0}\right)$.

\section{Simulation details}

Our calculations have been performed on a set of ensembles with $N_{\mathrm{f}}=2$ flavours of dynamical, mass-degenerate, $\mathrm{O}(a)$-improved Wilson quarks and the Wilson plaquette action. The improvement coefficient $c_{\mathrm{sw}}$ was tuned according to the non-perturbative determination of ref. [66]. The gauge configurations have been generated as part of the CLS (Coordinated Lattice Simulations) initiative, using the deflation-accelerated DD-HMC [67, 68] and MP-HMC [69] algorithms.

In table 1 we have compiled the parameter values, system sizes and overall statistics used in our determination of the hadronic vacuum polarization contribution. The values for the lattice scale reported in the table have been determined using the kaon decay constant $[70,71]$. In order to enhance statistics we have used four source positions per configuration, except for the most chiral ensembles G8 and O7 for which up to 16 different sources were chosen. The resulting number of measurements for each ensemble is shown in the right-most column of table 1 .

The bare values of the strange quark mass used in this work are based on an update of the analysis of ref. [70] where the physical values of the kaon mass and decay constant were used to set $\kappa_{s}$. The updated analysis [72] includes improved determinations of the 


\begin{tabular}{|ccccccccc|}
\hline Run & $L / a$ & $\beta$ & $\kappa$ & $m_{\pi} L$ & $a[\mathrm{fm}]$ & $m_{\pi}[\mathrm{MeV}]$ & $N_{\text {cfg }}$ & $N_{\text {meas }}$ \\
\hline A3 & 32 & 5.20 & 0.13580 & 6.0 & $0.0755(9)(7)$ & 495 & 251 & 1004 \\
A4 & 32 & 5.20 & 0.13590 & 4.7 & $0.0755(9)(7)$ & 381 & 400 & 1600 \\
A5 & 32 & 5.20 & 0.13594 & 4.0 & $0.0755(9)(7)$ & 331 & 251 & 1004 \\
B6 & 48 & 5.20 & 0.13597 & 5.0 & $0.0755(9)(7)$ & 281 & 306 & 1224 \\
\hline E5 & 32 & 5.30 & 0.13625 & 4.7 & $0.0658(7)(7)$ & 437 & 1000 & 4000 \\
F6 & 48 & 5.30 & 0.13635 & 5.0 & $0.0658(7)(7)$ & 311 & 300 & 1200 \\
F7 & 48 & 5.30 & 0.13638 & 4.2 & $0.0658(7)(7)$ & 265 & 250 & 1000 \\
G8 & 64 & 5.30 & 0.13642 & 4.0 & $0.0658(7)(7)$ & 185 & 325 & 4588 \\
\hline N5 & 48 & 5.50 & 0.13660 & 5.2 & $0.0486(4)(5)$ & 441 & 347 & 1388 \\
N6 & 48 & 5.50 & 0.13667 & 4.0 & $0.0486(4)(5)$ & 340 & 559 & 2236 \\
O7 & 64 & 5.50 & 0.13671 & 4.2 & $0.0486(4)(5)$ & 268 & 149 & 2384 \\
\hline
\end{tabular}

Table 1. Details of the lattice ensembles used in the calculation of the hadronic vacuum polarization, showing the lattice extent, $L$, where $T=2 L$, the values of the bare coupling $\beta$ and light quark hopping parameter $\kappa$ in the lattice action, as well as the lattice spacing and pion masses in physical units. $N_{\text {cfg }}$ and $N_{\text {meas }}$ denote the number of gauge configurations and measurements, respectively.

renormalization factors $Z_{\mathrm{A}}$ of the axial current, increased statistics, as well as a new measurement of $\kappa_{s}$ for the ensembles B6 and G8. In the charm sector, we used the bare quark masses determined from the experimental value of the $D_{s}$-meson mass in ref. [73] for the two finest values of the lattice spacing. Based on these results, at $\beta=5.2$ we estimated the hopping parameter $\kappa_{c}$ of the charm quark from the $a^{2}$ dependence of the ratio, $m_{c} / m_{s}$. Values for $\kappa_{s}$ and $\kappa_{c}$ are listed in table 2.

In our calculation we have considered a mixed vector correlator including the conserved point-split vector current

$$
V_{\mu, f}^{\mathrm{ps}}(x)=\frac{1}{2}\left(\bar{\psi}_{f}(x+a \hat{\mu})\left(1+\gamma_{\mu}\right) U_{\mu}^{\dagger}(x) \psi_{f}(x)-\bar{\psi}_{f}(x)\left(1-\gamma_{\mu}\right) U_{\mu}(x) \psi_{f}(x+a \hat{\mu})\right),
$$

and the local vector current

$$
V_{\mu, f}^{\mathrm{loc}}(x)=\bar{\psi}_{f}(x) \gamma_{\mu} \psi_{f}(x),
$$

where $f$ denotes one of the quark flavours $u, d, s$ and $c$. The local current is neither conserved nor improved, yet it can be renormalized in a fashion that is consistent with $\mathrm{O}(a)$ improvement [74]

$$
V_{\mu, f}^{\mathrm{R}}=Z_{\mathrm{V}}\left(1+b_{\mathrm{V}} a m_{f}\right)\left(V_{\mu, f}^{\mathrm{loc}}+a c_{\mathrm{V}} \partial_{\nu} T_{\mu \nu, f}\right) .
$$

Here $m_{f}$ denotes the bare subtracted quark mass of quark flavour $f, b_{\mathrm{V}}$ and $c_{\mathrm{V}}$ are improvement coefficients, and $T_{\mu \nu, f}(x)=-\bar{\psi}_{f}(x) \frac{1}{2}\left[\gamma_{\mu}, \gamma_{\nu}\right] \psi_{f}(x)$ is the tensor current. The conserved vector current, while not subject to renormalization, requires $\mathrm{O}(a)$ improvement even at tree level, which was not considered in this work. Since we did not determine the matrix elements containing the derivative of the tensor current, our results for $a_{\mu}^{\text {hvp }}$ are not fully $\mathrm{O}(a)$ improved.

In the light quark sector the mass-dependent factor in eq. (3.3) is usually a small correction. However, since we compute the contribution from the charm quark to $a_{\mu}^{\text {hvp }}$, the 
corresponding mass dependence is sizeable and must be included for a reliable extrapolation to the continuum limit. We have considered two different procedures for the determination of the renormalization factor of the local vector current, including the mass dependence:

1. Determine $Z_{\mathrm{V}}$ using the interpolating formula in ref. [75] and evaluate the one-loop expression for the improvement coefficient $b_{\mathrm{V}}$ from [76] using the boosted coupling $g^{2} \equiv g_{0}^{2} / \frac{1}{3} \operatorname{Tr}\left\langle U_{P}\right\rangle$.

2. Fix the (mass-dependent) renormalization factor $Z_{\mathrm{V}}^{\left(m_{f}\right)}$ of the local vector current from a ratio of two- and three-point correlation functions, where the latter involve the local current $V_{0, f}^{\text {loc }}$.

Details of the second procedure and a full set of results can be found in appendix A. For our main results reported in section 4 we have adopted $Z_{\mathrm{V}}^{\left(m_{f}\right)}$ as determined via the second procedure. As will be discussed in detail in section 5, we observe large lattice artefacts in the case of the charm quark contribution to $a_{\mu}^{\text {hvp }}$. In order to check for the stability of the continuum extrapolation we have compared the results obtained using both procedures to determine the current normalization and found very good agreement.

With the above definitions of the currents, the vacuum polarization tensor can be expressed in terms of the mixed vector correlator as

$$
\Pi_{\mu \nu}(\hat{Q})=a^{4} \sum_{f, f^{\prime}} q_{f} q_{f^{\prime}} Z_{\mathrm{V}}^{\left(m_{f^{\prime}}\right)} \sum_{x} \mathrm{e}^{i Q(x+a \hat{\mu} / 2)}\left\langle V_{\mu, f}^{\mathrm{ps}}(x) V_{\nu, f^{\prime}}^{\mathrm{loc}}(0)\right\rangle
$$

where $q_{f}, q_{f^{\prime}}$ denote the electric charges of quark flavours $f$ and $f^{\prime}$, and $\hat{Q}_{\mu}=\frac{2}{a} \sin \left(\frac{a Q_{\mu}}{2}\right)$ is the lattice momentum. Like in our previous publication [32] we have used twisted boundary conditions [61-63] in order to apply an additive shift to the momentum of the quark propagator. In this work we used a single value of the twist angle, chosen such as to provide three equidistant values of $Q^{2}$ between the lowest two Fourier momenta, as well as one additional data point below $(2 \pi / L)^{2}$. The imposition of twisted boundary conditions induces the breaking of isospin symmetry and modifies the Ward identity of the vacuum polarization tensor that guarantees its transversality [64]. We have checked explicitly [77] that the violation of the Ward identity has a negligible effect on the determination of $\Pi\left(Q^{2}\right)$.

It has been noted in [51, 78] (see also $[44,79]$ ) that the vacuum polarization tensor does not vanish at $Q=0$ in finite volume, $\Pi_{\mu \nu}(0) \neq 0$. In order to reduce finite-volume effects it is then advantageous to subtract the contribution $\Pi_{\mu \nu}(0)$, which is easily effected via a simple modification of the phase factor in eq. (3.4), i.e.

$$
\Pi_{\mu \nu}(\hat{Q})-\Pi_{\mu \nu}(\hat{0})=a^{4} \sum_{f, f^{\prime}} q_{f} q_{f^{\prime}} Z_{\mathrm{V}}^{\left(m_{f^{\prime}}\right)} \sum_{x}\left(\mathrm{e}^{i Q(x+a \hat{\mu} / 2)}-1\right)\left\langle V_{\mu, f}^{\mathrm{ps}}(x) V_{\nu, f^{\prime}}^{\mathrm{loc}}(0)\right\rangle .
$$

In addition to computing $\Pi_{\mu \nu}(Q)$ we have also considered the spatially summed vector correlator, given by

$$
G\left(x_{0}\right) \delta_{k l}=-a^{3} \sum_{f, f^{\prime}} q_{f} q_{f^{\prime}} Z_{\mathrm{V}}^{\left(m_{f^{\prime}}\right)} \sum_{\vec{x}}\left\langle V_{k, f}^{\mathrm{ps}}(x) V_{l, f^{\prime}}^{\mathrm{loc}}(0)\right\rangle .
$$




\begin{tabular}{|cccc|cc|cc|}
\hline Run & $a m_{\pi}$ & $a m_{\rho}$ & $m_{\pi} / m_{\rho}$ & $\kappa_{s}$ & $a m_{V}(s \bar{s})$ & $\kappa_{c}$ & $a m_{V}(c \bar{c})$ \\
\hline A3 & $0.1893(6)$ & $0.3937(29)$ & $0.481(4)$ & 0.135364355 & $0.4399(22)$ & 0.12552 & $1.1719(6)$ \\
A4 & $0.1459(7)$ & $0.3619(31)$ & $0.403(3)$ & 0.135303471 & $0.4291(15)$ & 0.12525 & $1.1816(5)$ \\
A5 & $0.1265(8)$ & $0.3490(41)$ & $0.363(5)$ & 0.135275643 & $0.4259(26)$ & 0.12515 & $1.1848(7)$ \\
B6 & $0.1073(7)$ & $0.3265(82)$ & $0.328(9)$ & 0.135257096 & $0.4133(22)$ & 0.12506 & $1.1831(8)$ \\
\hline E5 & $0.1458(3)$ & $0.3208(29)$ & $0.455(4)$ & 0.135802302 & $0.3704(13)$ & 0.12724 & $1.0264(3)$ \\
F6 & $0.1036(3)$ & $0.2928(38)$ & $0.354(5)$ & 0.135766419 & $0.3624(17)$ & 0.12713 & $1.0295(5)$ \\
F7 & $0.0885(3)$ & $0.2779(49)$ & $0.318(6)$ & 0.135755498 & $0.3546(18)$ & 0.12713 & $1.0272(5)$ \\
G8 & $0.0617(3)$ & $0.2578(39)$ & $0.239(4)$ & 0.135740236 & $0.3503(20)$ & 0.12710 & $1.0280(6)$ \\
\hline N5 & $0.1086(2)$ & $0.2331(27)$ & $0.466(5)$ & 0.136275891 & $0.2727(15)$ & 0.13026 & $0.7628(3)$ \\
N6 & $0.0838(2)$ & $0.2244(28)$ & $0.374(5)$ & 0.136263492 & $0.2710(09)$ & 0.13026 & $0.7611(3)$ \\
O7 & $0.0660(1)$ & $0.2172(77)$ & $0.304(11)$ & 0.136256771 & $0.2664(17)$ & 0.13022 & $0.7621(5)$ \\
\hline
\end{tabular}

Table 2. Masses of the pion, the $\rho$-meson masses, as well as the $s \bar{s}$ and $c \bar{c}$ vector states as determined from single exponential fits.

The sum $\sum_{f, f^{\prime}} \ldots$ in equations (3.4) and (3.6) runs over all quark flavours included in the electromagnetic currents. Here we focus on the quark-connected contributions to the vector correlator. In order to quantify individual flavour contributions to $a_{\mu}^{\text {hvp }}$ it is useful to define

$$
G^{f}\left(x_{0}\right)=-\frac{a^{3}}{3} \sum_{k=1}^{3} \sum_{\vec{x}} q_{f}^{2} Z_{\mathrm{V}}^{\left(m_{f}\right)}\left\langle V_{k, f}^{\mathrm{ps}}\left(x_{0}, \vec{x}\right) V_{k, f}^{\mathrm{loc}}(0)\right\rangle, \quad f=(u d), s, c, \ldots,
$$

where $q_{u d}^{2}=5 / 9$, and it is understood that the expectation value is restricted to quarkconnected diagrams. The vector correlator in the long-distance regime is constrained by the mass spectrum of the theory. Depending on the value of the light quark mass on a given ensemble, the lowest-lying state corresponds either to the vector meson or to a twopion state. For a reliable determination of the energy levels in the vector channel, we have computed additional correlators using standard Gaussian smearing [80] in the calculation of quark propagators, with APE-smeared link variables [81] in the spatial directions. The mass in the vector channel and also the pion mass used in this study were determined from the appropriate correlation functions with smearing applied both at the source and sink. The corresponding mass estimates are listed in table 2.

All statistical errors were estimated using a bootstrap procedure with 10,000 samples. For the estimation of systematic errors we employed the so-called "extended frequentist method" $[82,83]$ and determined the distributions of results obtained from a set of variations of our analysis procedure. Details are provided in the sections describing our results.

\section{Calculation of $a_{\mu}^{\text {hvp }}$}

In this section we report on the determination of $a_{\mu}^{\text {hvp }}$ for all our ensembles, employing different methods, in order to check for systematic effects. 


\section{1 $a_{\mu}^{\text {hvp }}$ from the hybrid method}

Our calculation of $a_{\mu}^{\text {hvp }}$ from the vacuum polarization tensor proceeds by evaluating the vacuum-subtracted tensor defined in eq. (3.5) and factoring out the tensor structure according to eq. (2.3). In order to determine the additive renormalization $\Pi(0)$ and describe the data in the small momentum regime, we have employed the ansatz

$$
\Pi\left(Q^{2}\right)=\Pi(0)+P_{[n, m]}\left(Q^{2}\right),
$$

where $P_{[n, m]}$ denotes the Padé approximant of order $[n, m]$. Following ref. [52] we consider $n=m$ or $n=m+1$ and write $P_{[n, m]}$ as

$$
P_{[n, m]}\left(Q^{2}\right)=Q^{2}\left\{A_{0} \delta_{n, m+1}+\sum_{k=1}^{m} \frac{A_{k}}{B_{k}+Q^{2}}\right\} .
$$

In accordance with the discussion of the "hybrid strategy" in [56] the main task is to determine the Padé representation in an interval $0<Q^{2} \lesssim Q_{\text {cut }}^{2}$. Here we have adopted two procedures: the first proceeds by determining the coefficients $A_{k}$ and $B_{k}$ from fits to the VPF, the second uses time moments to construct the Padé approximation for $0<Q^{2} \lesssim Q_{\text {cut }}^{2}$.

Ideally, the Padé representation of $\Pi\left(Q^{2}\right)$ should be constructed by considering a sequence of approximants of increasing order [52]. However, when confronted with actual simulation data one often finds that the latter are not constraining enough to allow for a systematic investigation whether successive Padés converge towards the actual VPF. One therefore resorts to constructing low-order Padé approximations, i.e. one-pole ansätze that are not much different from a vector meson dominance description. To minimize the bias incurred from using a particular Padé approximant, the value of $Q_{\text {cut }}^{2}$ should be chosen much smaller than $m_{\rho}^{2}$. However, one has to balance this requirement against fit stability and statistical accuracy. In order to have sufficiently many data points available so that stable correlated fits with acceptable $\chi^{2} /$ dof can be performed, we have chosen $Q_{\text {cut }}^{2} \approx 0.5 \mathrm{GeV}^{2}$. At our level of statistical precision we find that the data are well described by a Padé $[1,1]$ ansatz and exhibit values of the correlated $\chi^{2} /$ dof of order unity, except for ensembles E5 and N6 for which $\chi^{2} /$ dof $>6$. Using a Padé $[2,1]$ ansatz gave consistent results but larger statistical errors.

In order to calculate the light quark contribution to the anomalous magnetic moment, $\left(a_{\mu}^{\text {hvp }}\right)^{u d}$, we have evaluated the convolution integral of eq. (2.1) in the interval $0 \leq Q^{2} \leq$ $Q_{\text {cut }}^{2}$ by inserting $\Pi\left(Q^{2}\right)^{u d}-\Pi(0)^{u d}$ as determined by the Padé $[1,1]$ fit. The contribution from the region $Q^{2}>Q_{\text {cut }}^{2}$ was computed using trapezoidal integration, and the resulting values of $\left(a_{\mu}^{\text {hvp }}\right)^{u d}$ are shown in the third column of table 3 . To check for stability against variation of the scale $Q_{\text {cut }}$ we have computed $\left(a_{\mu}^{\text {hvp }}\right)^{u d}$ for $Q_{\text {cut }}^{2} \approx 0.3-0.35 \mathrm{GeV}^{2}$. We find agreement within slightly larger errors with the numbers reported in table 3 .

For the determination of the strange quark contribution to the vacuum polarization, $\Pi\left(Q^{2}\right)^{s}-\Pi(0)^{s}$, and the anomalous magnetic moment, $\left(a_{\mu}^{\mathrm{hvp}}\right)^{s}$, we have followed the same procedures as for $\left(a_{\mu}^{\mathrm{hvp}}\right)^{u d}$. Concerning the influence of variations in the value of $Q_{\text {cut }}^{2}$ and the order of the Padé ansatz we came to the same conclusions. The results for $\left(a_{\mu}^{\text {hvp }}\right)^{s}$ 


\begin{tabular}{|c|ccc|cc|}
\hline Run & $Q_{\text {cut }}^{2}\left[\mathrm{GeV}^{2}\right]$ & $\left(a_{\mu}^{\mathrm{hvp}}\right)^{u d}$ & $\left(a_{\mu}^{\mathrm{hvp}}\right)^{s}$ & $Q_{\text {cut }}^{2}\left[\mathrm{GeV}^{2}\right]$ & $\left(a_{\mu}^{\mathrm{hvp}}\right)^{c}$ \\
\hline A3 & 0.484 & $272(09)$ & $40.4(6)$ & 0.222 & $7.6(4)$ \\
A4 & 0.484 & $345(14)$ & $41.9(5)$ & 0.222 & $7.1(3)$ \\
A5 & 0.484 & $357(32)$ & $43.0(7)$ & 0.397 & $6.7(1)$ \\
B6 & 0.501 & $386(08)$ & $44.0(3)$ & 0.146 & $7.2(3)$ \\
\hline E5 & 0.522 & $326(09)^{*}$ & $44.2(6)^{*}$ & 0.364 & $7.9(1)$ \\
F6 & 0.500 & $390(10)$ & $46.1(3)$ & 0.192 & $7.8(2)$ \\
F7 & 0.500 & $459(17)$ & $46.8(4)$ & 0.245 & $8.1(1)$ \\
G8 & 0.499 & $504(10)$ & $47.5(4)$ & 0.138 & $8.1(3)$ \\
\hline N5 & 0.497 & $321(11)$ & $43.5(6)$ & 0.282 & $9.4(2)$ \\
N6 & 0.497 & $373(18)^{*}$ & $46.9(5)$ & 0.353 & $9.4(1)$ \\
O7 & 0.496 & $421(11)$ & $47.6(4)$ & 0.253 & $9.4(2)$ \\
\hline
\end{tabular}

Table 3. Results for the hadronic vacuum polarization contributions to the muon anomalous magnetic moment (in units of $10^{-10}$ ) from the light, strange and charm flavours, determined via the hybrid method, where the low-momentum representation of the VPF was determined from a fit. Results marked by an asterisk are associated with unacceptably large values of $\chi^{2} /$ dof (see text).

determined for $Q_{\text {cut }}^{2} \approx 0.5 \mathrm{GeV}^{2}$ are listed in the fourth column of table 3. For ensemble E5 we again found $\chi^{2} /$ dof $\approx 7$, both for the Padé $[1,1]$ and $[2,1]$ fits. The corresponding entry is marked by an asterisk in table 3 and is excluded from the subsequent analysis.

The $Q^{2}$-dependence of the charm quark contribution to $\Pi\left(Q^{2}\right)$ shows a lot less curvature compared to the lighter flavours. We have therefore applied a slightly different procedure, by fitting $\Pi\left(Q^{2}\right)$ not only to a Padé $[1,1]$ ansatz but also to a linear function in $Q^{2}$. Starting from $Q_{\text {cut }}^{2} \approx 0.5 \mathrm{GeV}^{2}$ we have gradually lowered $Q_{\text {cut }}^{2}$ until the two different ansätze gave consistent results. The corresponding estimates of $\left(a_{\mu}^{\text {hvp }}\right)^{c}$ are listed alongside with the respective values of $Q_{\text {cut }}^{2}$ in table 3. A striking but not unexpected feature of $\left(a_{\mu}^{\text {hvp }}\right)^{c}$ is the strong dependence on the lattice spacing. This is seen easily by comparing the estimates for $\left(a_{\mu}^{\text {hvp }}\right)^{c}$ for ensembles B6, F7 and O7: at approximately constant pion mass in physical units the results for $\left(a_{\mu}^{\text {hvp }}\right)^{c}$ vary by $30-40 \%$ within the range of lattice spacings considered in this work.

An alternative determination of the low-momentum representation of $\hat{\Pi}\left(Q^{2}\right)$ is achieved by computing time moments of the vector correlator. These are linked to the coefficients $\Pi_{j}$ in the Taylor-series expansion of the vacuum polarization function and also to the additive renormalization $\Pi(0)$ (see eq. (2.12)). The $\Pi_{j}$ 's can then be used to construct the coefficients $A_{k}, B_{k}$ in the Padé representation of eq. (4.2). For instance, the Padé $[1,1]$ approximant written in terms of the expansion coefficients reads

$$
P_{[1,1]}\left(Q^{2}\right)=Q^{2} \frac{\Pi_{1}^{2}}{\Pi_{1}-\Pi_{2} Q^{2}},
$$




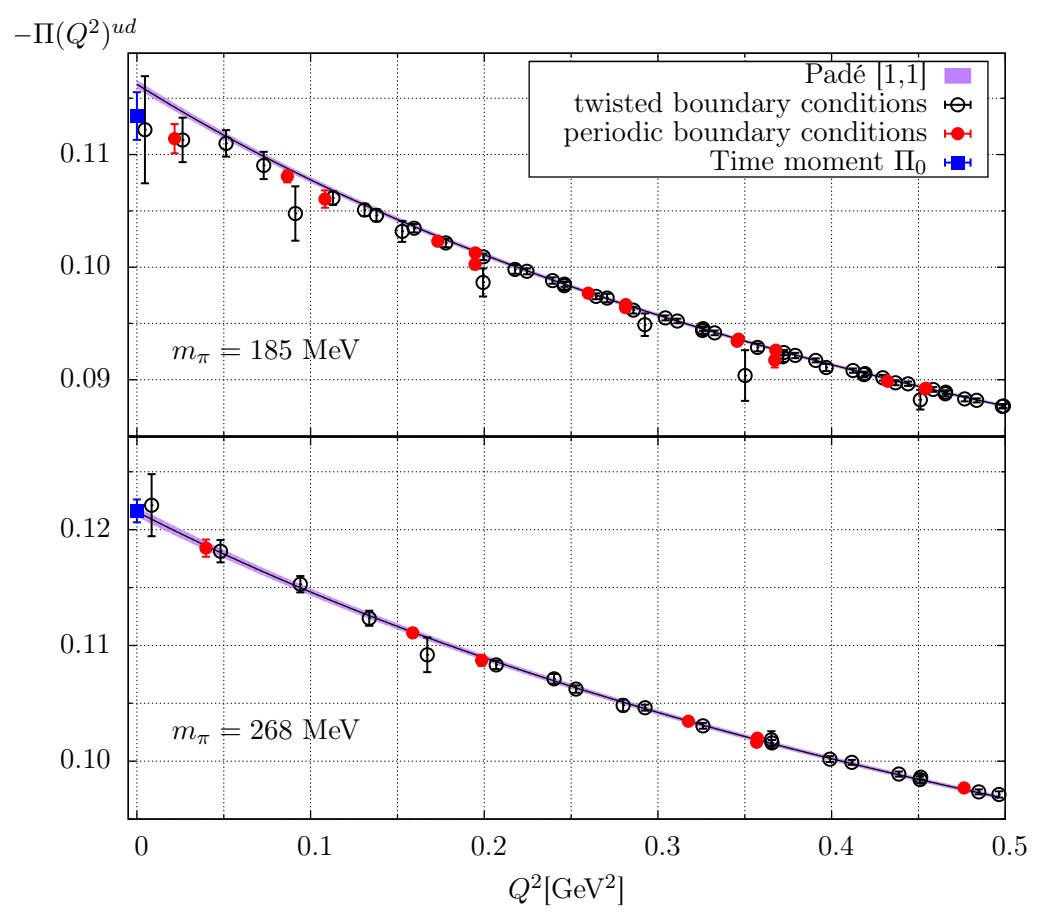

Figure 1. The $u, d$ contributions to the vacuum polarization function in the range $0<Q^{2} \leq$ $0.5 \mathrm{GeV}^{2}$ for ensembles G8 (top) and O7 (bottom). Data points corresponding to Fourier momenta are represented by filled red circles, while open black circles denote data points computed using twisted boundary conditions. The curves represent the fits using a Padé approximant of order $[1,1]$. Blue filled squares indicate the value of $\Pi(0)$ determined from the second time moment.

and expressions for higher-order Padés can easily be worked out. The determination of the time moments proceeds by summing the vector correlator over all Euclidean times. As in the case of the TMR, which is discussed in detail in the next subsection, this requires some sort of modelling of the long-distance regime of $G^{f}\left(x_{0}\right)$. To this end we have assumed that $G^{f}\left(x_{0}\right)$ is described by a single exponential for $x_{0}>x_{0}^{\text {cut }}$ (see eq. (4.6) below). A more detailed discussion is presented in section 4.2.

It is instructive to compare the Padé representation of $\Pi\left(Q^{2}\right)$ as determined from time moments to that obtained from fits to $\Pi\left(Q^{2}\right)$ below $Q_{\text {cut }}^{2}$ discussed earlier. Such a comparison is shown in figure 1 for the ensembles G8 and O7. In particular, we compare the intercept $\Pi(0)^{u d}$ as obtained from a Padé $[1,1]$ fit for $0<Q^{2} \leq 0.5 \mathrm{GeV}^{2}$ to its determination from the second time moment. As is apparent from the figure the two procedures agree very well, which is an important cross check. Typically, the estimate of $\Pi(0)^{u d}$ from the fit has a smaller error. Having computed the coefficients $\Pi_{0}, \Pi_{1}, \ldots, \Pi_{4}$ from time moments we constructed the Padé $[1,1]$ and $[2,1]$ representations of $\hat{\Pi}\left(Q^{2}\right)$ in the interval $0 \leq Q^{2} \leq Q_{\text {cut }}^{2}$. As before we determined $a_{\mu}^{\text {hvp }}$ by performing the convolution integral over $\hat{\Pi}\left(Q^{2}\right)$ for $Q^{2}>Q_{\text {cut }}^{2}$ using trapezoidal integration. Thus, our way of employing time moments differs from the procedures applied in refs. [34, 39], where the subtracted vacuum polarization function $\hat{\Pi}\left(Q^{2}\right)$ is constructed from time moments within the entire momentum interval. 


\begin{tabular}{|c|cc|cc|cc|}
\hline Run & $Q_{\text {cut }}^{2}\left[\mathrm{GeV}^{2}\right]$ & $\left(a_{\mu}^{\mathrm{hvp}}\right)^{u d}$ & $Q_{\text {cut }}^{2}\left[\mathrm{GeV}^{2}\right]$ & $\left(a_{\mu}^{\mathrm{hvp}}\right)^{s}$ & $Q_{\text {cut }}^{2}\left[\mathrm{GeV}^{2}\right]$ & $\left(a_{\mu}^{\mathrm{hvp}}\right)^{c}$ \\
\hline A3 & 0.263 & $287(3)$ & 0.328 & $42.8(3)$ & 0.156 & $8.7(3)$ \\
A4 & 0.222 & $354(3)$ & 0.328 & $44.7(3)$ & 0.156 & $8.3(3)$ \\
A5 & 0.277 & $360(7)$ & 0.263 & $44.7(4)$ & 0.156 & $8.1(4)$ \\
B6 & 0.152 & $410(8)$ & 0.394 & $46.6(3)$ & 0.123 & $7.9(6)$ \\
\hline E5 & 0.451 & $319(3)$ & 0.451 & $45.1(2)$ & 0.105 & $9.0(4)$ \\
F6 & 0.470 & $397(5)$ & 0.233 & $47.4(4)$ & 0.130 & $8.8(4)$ \\
F7 & 0.346 & $478(9)$ & 0.245 & $48.4(4)$ & 0.154 & $9.0(4)$ \\
G8 & 0.195 & $497(7)$ & 0.138 & $49.5(7)$ & 0.304 & $9.1(1)$ \\
\hline N5 & 0.238 & $327(3)$ & 0.497 & $45.1(3)$ & 0.282 & $10.3(1)$ \\
N6 & 0.497 & $377(4)$ & 0.427 & $47.5(2)$ & 0.238 & $10.4(1)$ \\
O7 & 0.365 & $427(11)$ & 0.451 & $48.8(4)$ & 0.167 & $10.2(4)$ \\
\hline
\end{tabular}

Table 4. Results for the various flavour contributions to $a_{\mu}^{\text {hvp }}$ (in units of $10^{-10}$ ) determined via the hybrid method. For $Q^{2}<Q_{\text {cut }}^{2}$ the VPF is represented by a Padé [1,1] constructed from the time moments.

In order to guarantee a smooth transition between the low-momentum representation and the actual data for $\hat{\Pi}\left(Q^{2}\right)=4 \pi^{2}\left(\Pi\left(Q^{2}\right)-\Pi_{0}\right)$ we have chosen $Q_{\text {cut }}^{2}$ so as to minimize the difference between the Padé approximation of $\hat{\Pi}\left(Q^{2}\right)$ and the data within the interval $Q^{2}=0.1-0.5 \mathrm{GeV}^{2}$. Results for $a_{\mu}^{\text {hvp }}$ obtained via this procedure are listed in table 4 . We found the differences between the Padé $[1,1]$ and $[2,1]$ descriptions of the low- $Q^{2}$ regime to be negligible.

\subsection{The TMR method for $a_{\mu}^{\text {hvp }}$}

The integral representation of the subtracted vacuum polarization function, $\hat{\Pi}\left(Q^{2}\right)$, is shown in eq. (2.5), and the hadronic vacuum polarization contribution of quark flavour $f=$ $(u d), s, c$ to $a_{\mu}$ is then obtained as [51],

$$
\left(a_{\mu}^{\mathrm{hvp}}\right)^{f}=\left(\frac{\alpha}{\pi}\right)^{2} \int_{0}^{\infty} d x_{0} G^{f}\left(x_{0}\right) \widetilde{K}\left(x_{0} ; m_{\mu}\right) .
$$

In appendix B we derive an explicit expression which describes $\widetilde{K}\left(x_{0}, m_{\mu}\right)$ with an accuracy of $\mathrm{O}\left(10^{-6}\right)$. The kernel is proportional to $x_{0}^{4}$ at small $x_{0}$, and to $x_{0}^{2}$ at large $x_{0}$. The integration must be performed over all Euclidean times $x_{0}$, and thus the challenge in this method is to control the long-distance behaviour of the spatially summed vector correlator $G^{f}\left(x_{0}\right)$ defined in eq. (3.7). The main issues are that

(a) the relative error of $G^{f}\left(x_{0}\right)$ increases at large $x_{0}$,

(b) the lattice extent is finite in the time direction, and

(c) the tail of the correlator is most affected by the finite spatial size of the box $L$. 
In order to handle the large- $x_{0}$ part separately, we define our estimator

$$
G^{f}\left(x_{0}\right)=\left\{\begin{array}{l}
G^{f}\left(x_{0}\right)_{\text {inter }} x_{0} \leq x_{0}^{\text {cut }}, \\
G^{f}\left(x_{0}\right)_{\text {ext }} \quad x_{0}>x_{0}^{\text {cut }} .
\end{array}\right.
$$

The subscript "inter" denotes that the vector correlator has been obtained from a local cubic spline interpolation of the numerical data. The long-distance part $G^{f}\left(x_{0}\right)_{\text {ext }}$ is obtained by extending the correlator by one of the methods specified below.

Items (a) and (b) can be dealt with by extrapolating the correlator using a sum of exponentials. Indeed, in a finite volume, the spectral representation implies that the correlator is exactly given by an infinite sum of exponentials $\exp \left(-E_{n} x_{0}\right)$. The lowest few energyeigenstates ${ }^{1}$ dominate at large $x_{0}$. Therefore the simplest incarnation of this method is to use a single-exponential extension of the correlator,

$$
G^{f}\left(x_{0}\right)_{\mathrm{ext}}=A e^{-m_{V} x_{0}}, \quad x_{0}>x_{0}^{\text {cut }} .
$$

The parameters $\left(A, m_{V}\right)$ depend on the flavour composition $f=(u d), s, c$ of the vector current. Clearly, the systematic error incurred by using a single exponential must be investigated. Since the energy levels only depend on the quantum numbers of the interpolating operator, they can also be determined from auxiliary correlation functions. In our benchmark analysis, whose preliminary results have been presented in [84], we extract $m_{V}$ from the two-point function of a smeared vector operator, obtaining the masses reported in table 2. The amplitudes $A$ are then determined from a one-parameter fit to eq. (4.6) using these masses as input. A compilation of results for $a_{\mu}^{\text {hvp }}$ extracted via the TMR is shown in table 5 along with the respective values of $x_{0}^{\text {cut }}$. As an illustration of the method, we plot the integrand of eq. (4.4) for the light-quark connected contribution on the two ensembles with the lightest pion masses, G8 and O7, in figure 2. The extension method just described is labelled as '1-exp'. Various coloured bands represent other methods (discussed below) to constrain the long-distance behaviour of the vector correlator.

The choice of $x_{0}^{\text {cut }}$ affects the accuracy of $a_{\mu}^{\text {hvp }}$ since larger values of $x_{0}^{\text {cut }}$ increase the statistical error because of the quickly rising noise-to-signal ratio in the correlator data. By contrast, a smaller cutoff implies that estimates of $a_{\mu}^{\text {hvp }}$ will be more strongly affected by systematic effects arising from assumptions regarding the asymptotic behaviour of the correlator. We have chosen $x_{0}^{\text {cut }}$ as the value beyond which the statistical signal deteriorates to such an extent that the original data do not accurately constrain the correlator anymore. In terms of statistical accuracy this represents the most conservative choice, since the interpolation of $G^{f}\left(x_{0}\right)$ is used within the maximum Euclidean time range where the signal is not lost. We have checked explicitly that our estimates are not affected by the particular choice of $x_{0}^{\text {cut }}$. Moreover, in the case of the strange and charm quark contributions we have found that the correlators fall off so rapidly that the effect of truncating the integral in eq. (4.4) at $x_{0}=x_{0}^{\text {cut }}$ on the estimates of $\left(a_{\mu}^{\text {hvp }}\right)^{s}$ and $\left(a_{\mu}^{\text {hvp }}\right)^{c}$ is insignificant. We conclude that in this case the systematic error arising from the modelling of the long-distance contribution is negligible for $x_{0}^{\text {cut }} \gtrsim 1.2 \mathrm{fm}$. In the future, variance-reduction strategies, such as

\footnotetext{
${ }^{1}$ These states belong to the irreducible representation $T_{1}$ of the cubic group.
} 


\begin{tabular}{|ccccccc|}
\hline Run & $x_{0}^{\text {cut }}[\mathrm{fm}]$ & $\left(a_{\mu}^{\mathrm{hvp}}\right)_{1-\exp }^{u d}$ & $\left(a_{\mu}^{\mathrm{hvp}}\right)_{\mathrm{GS}}^{u d}$ & $\left(a_{\mu}^{\mathrm{hvp}}\right)_{\mathrm{GS}, \text { inf }}^{u d}$ & $\left(a_{\mu}^{\mathrm{hvp}}\right)_{1-\exp }^{s}$ & $\left(a_{\mu}^{\mathrm{hvp}}\right)_{1-\exp }^{c}$ \\
\hline A3 & 1.13 & $278(04)$ & & & $41.8(4)$ & $8.05(4)$ \\
A4 & 1.13 & $342(06)$ & & & $43.5(3)$ & $7.78(3)$ \\
A5 & 1.13 & $350(16)$ & $347(14)$ & $355(14)$ & $43.6(4)$ & $7.56(4)$ \\
B6 & 1.13 & $397(12)$ & $403(13)$ & $407(13)$ & $45.3(4)$ & $7.52(5)$ \\
\hline E5 & 1.38 & $314(04)$ & & & $44.7(2)$ & $9.28(2)$ \\
F6 & 1.38 & $392(10)$ & $392(11)$ & $395(11)$ & $47.1(4)$ & $9.15(3)$ \\
F7 & 1.38 & $469(17)$ & $474(18)$ & $481(18)$ & $48.0(4)$ & $9.17(4)$ \\
G8 & $1.32 / 1.18$ & $477(12)$ & $506(07)$ & $521(07)$ & $49.0(5)$ & $9.18(4)$ \\
\hline N5 & 1.17 & $323(05)$ & & & $44.7(4)$ & $10.49(3)$ \\
N6 & 1.17 & $372(08)$ & $373(05)$ & $383(04)$ & $47.0(3)$ & $10.57(2)$ \\
O7 & 1.17 & $420(13)$ & $428(07)$ & $436(07)$ & $48.2(5)$ & $10.45(5)$ \\
\hline
\end{tabular}

Table 5. Results for $a_{\mu}^{\text {hvp }}$ in units of $10^{-10}$ determined from the time-momentum representation along with the Euclidean time $x_{0}^{\text {cut }}$ that marks the switch from a cubic spline interpolation of the correlator to its long-distance representation. The label "1-exp" refers to the single exponential of eq. (4.6), while "GS" and "GS, inf" refer to the Gounaris-Sakurai-based extensions in finite and infinite volume, respectively. For the latter a slightly smaller value of $x_{0}^{\text {cut }}$ was used on ensemble G8 to stabilize the fit. At heavy pion mass only the one-exponential extension was considered.

those described in $[85,86]$ may be used to suppress the strong growth of the noise-to-signal ratio of $G^{f}\left(x_{0}\right)$, thereby reducing the need for modelling the large- $x_{0}$ behaviour.

We now return to the issue of the extension of the correlator $G^{u d}\left(x_{0}\right)$. On all our ensembles except for G8, a single exponential already provides a remarkably good description of the correlator for $x_{0} \geq x_{0}^{\text {cut }}$. The reason is that the lightest energy-eigenstate in the box has a large amplitude relative to the other states. This fact is well understood: the finite-volume energies and amplitudes are directly related to the timelike pion form factor $[87,88]$. The latter peaks at the $\rho$-resonance, $E=m_{\rho}$, and one state in the finite box almost always lies nearby in energy. It happens to be the lightest state on all but one ensemble. Thus the reason that the light-quark correlator $G^{u d}\left(x_{0}\right)$ is dominated by a single exponential is closely related to the ideas underlying the vector-meson dominance model (VMD) used in hadron phenomenology.

Obviously, the one-exponential extension has its limitations. This becomes most evident on ensemble G8, where one expects to find, below the energy level $E_{2}$ associated with a large amplitude, an energy level $E_{1}<E_{2}$ with a smaller amplitude. This conclusion is easily reached by initially neglecting the interactions between two pions in the $T_{1}$ representation, $E_{\pi \pi} \equiv E_{1}=2 \sqrt{m_{\pi}^{2}+(2 \pi / L)^{2}}(\approx 695 \mathrm{MeV}$ on G8). The non-vanishing scattering phase leads to a modest shift of the energy level. Obviously the result for $a_{\mu}^{\text {hvp }}$ incurs a bias if one ignores this low-lying state, but it is difficult to determine its precise energy and amplitude from $G^{u d}\left(x_{0}\right)$, because the amplitude is small. These observations also show that the finite-volume correlator behaves drastically differently at large $x_{0}$ than 


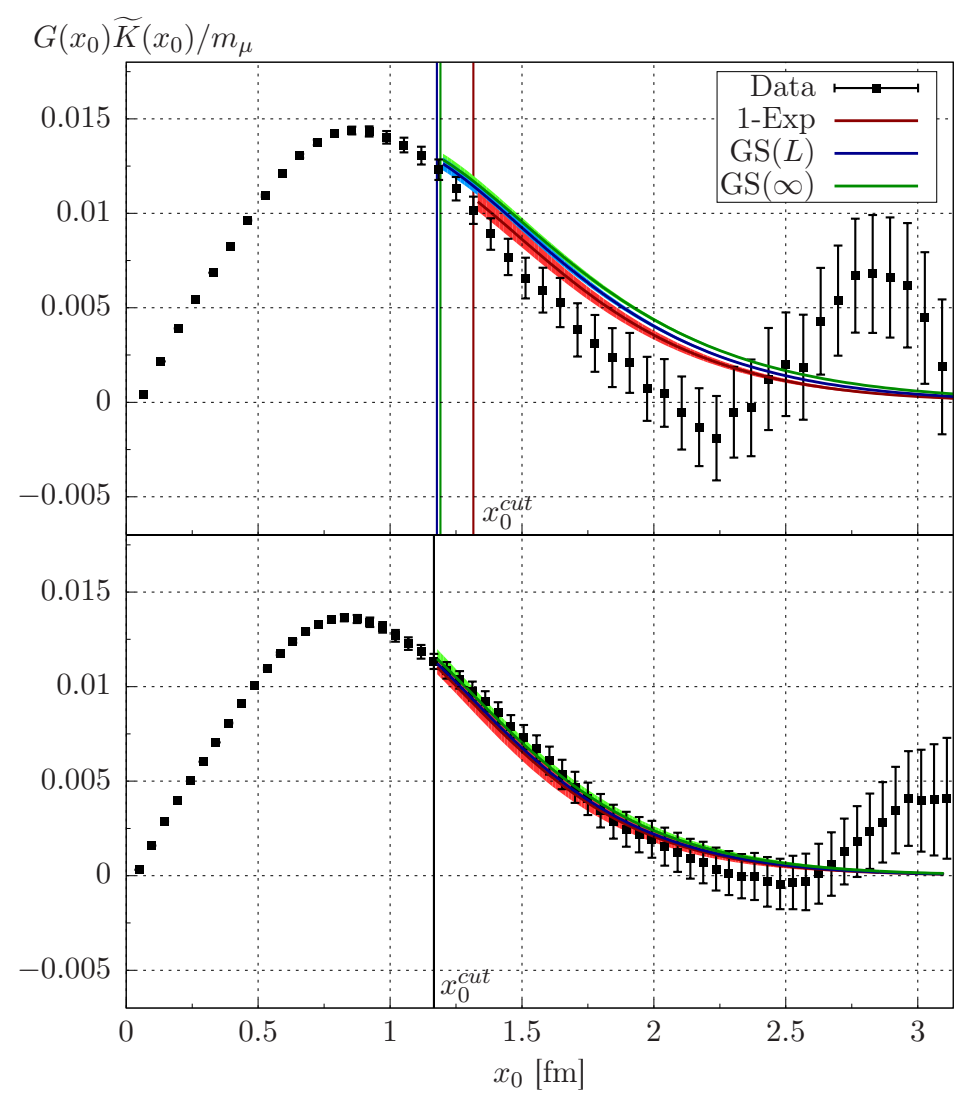

Figure 2. Data for the light quark contribution to the integrand $\widetilde{K}\left(x_{0} ; m_{\mu}\right) G^{u d}\left(x_{0}\right)$, scaled in units of the muon mass for ensembles G8 (top) and O7 (below). The coloured bands, which show the various methods to constrain the long-distance behaviour, start at the respective value of $x_{0}^{\text {cut }}$ as indicated by the vertical lines.

in infinite volume: in the latter case, $G^{u d}\left(x_{0}\right)$ is dominated by a two-pion continuum starting at $E=2 m_{\pi}(\approx 370 \mathrm{MeV}$ on G8) rather than by discrete energy levels. Thus the issue of extending the correlator $G^{u d}\left(x_{0}\right)$ to long distances is intimately related to the question of the finite-size effects on lattice determinations of $a_{\mu}^{\text {hvp }}$ (see item (c) above).

To prepare for a more sophisticated treatment of the long-distance behaviour of the vector correlator, it is useful to recall the isospin decomposition of eq. (2.15), i.e. $G\left(x_{0}\right)=$ $G^{\rho \rho}\left(x_{0}\right)+G^{I=0}\left(x_{0}\right)$. The iso-vector part $G^{\rho \rho}$ is directly proportional to the quark-connected light-quark contribution $G^{u d}$, i.e.

$$
G^{\rho \rho}\left(x_{0}\right)=\frac{9}{10} G^{u d}\left(x_{0}\right) .
$$

The $\omega$-resonance is the lowest-lying state in the iso-scalar channel, which has a much smaller width compared to the $\rho$. In particular, the decay of the $\omega$ into three pions is strongly suppressed, and thus the single exponential

$$
G^{I=0}\left(x_{0}\right) \propto \mathrm{e}^{-m_{\omega} x_{0}}
$$


is a good approximation for evaluating the iso-scalar contribution to the convolution integral in eq. (2.7). By exploiting the fact that the $\rho-\omega$ splitting is small, we arrive at our final ansatz for the long-distance contribution to the quark-connected light quark vector correlator, i.e.

$$
G^{u d}\left(x_{0}\right)_{\mathrm{ext}}=G^{\rho \rho}\left(x_{0}\right)_{\mathrm{ext}}+\frac{1}{10} G^{u d}\left(x_{0}\right)_{1-\exp } .
$$

In other words, we replace the light iso-scalar correlator by a single exponential with $m_{V}=m_{\rho}$ in the long-distance regime. ${ }^{2}$ In the following subsection we describe how $G^{\rho \rho}\left(x_{0}\right)_{\text {ext }}$ can be constrained via the Gounaris-Sakurai model.

\subsection{Gounaris-Sakurai based extension of the vector correlator}

As already advocated in [51], the calculation of the vector correlator for $a_{\mu}^{\text {hvp }}$ should ideally be accompanied by a dedicated study of the timelike pion form factor $F_{\pi}(\omega)$. This has been the subject of a few recent lattice calculations [89-91]. With the pion form factor at hand, the long-distance part of the iso-vector correlator $G^{\rho \rho}\left(x_{0}\right)_{\text {ext }}$ can be obtained straightforwardly. Moreover, one can compute the infinite-volume iso-vector correlator via

$$
G^{\rho \rho}\left(x_{0}\right)_{\mathrm{ext}}=\int_{0}^{\infty} d \omega \omega^{2} \rho\left(\omega^{2}\right) e^{-\omega x_{0}}, \quad \rho\left(\omega^{2}\right)=\frac{1}{48 \pi^{2}}\left(1-\frac{4 m_{\pi}^{2}}{\omega^{2}}\right)^{\frac{3}{2}}\left|F_{\pi}(\omega)\right|^{2},
$$

thus correcting model-independently for the dominant finite-size effects in $a_{\mu}^{\text {hvp }}$. Eq. (4.10) assumes that the $2 \pi$ channel saturates the iso-vector correlator, which is a good approximation if $x_{0}^{\text {cut }}$ is sufficiently large. However, lacking a full-scale calculation of the timelike pion form factor, we apply a simplified version of this strategy (at the cost of a certain modeldependence). Based on the success of the Gounaris-Sakurai (GS) model [92] in describing experimental data for $e^{+} e^{-} \rightarrow \pi^{+} \pi^{-}$data, we assume that the timelike pion form factor is well approximated by this model at the pion masses used in our ensembles. Since the GS model only contains two parameters (the $\rho$-mass and its width $\Gamma_{\rho}$ ), the same number as the one-exponential ansatz eq. (4.6), this simplified approach allows us to go beyond the one-exponential extension whilst remaining numerically viable given the available lattice data. The procedure can be summarized as follows:

1. Fix the GS parameter $m_{\rho}$ by identifying it with one of the energy levels determined from the smeared-smeared correlator.

2. Determine the GS parameter $\Gamma_{\rho}$ from the iso-vector correlator $G^{\rho \rho}\left(x_{0}\right)$, using $m_{\rho}$ as input.

3. Determine the low-lying energy levels and their amplitudes using the GS model and the Lüscher formalism. The finite-volume correlator can then be computed beyond $x_{0}^{\text {cut }}$ as the sum of the corresponding exponentials, and from there $\left(a_{\mu}^{\text {hvp }}\right)^{u d}$ is obtained.

\footnotetext{
${ }^{2}$ The iso-scalar contribution, $G^{I=0}\left(x_{0}\right)$, to which the second term in eq. (4.9) belongs, will be analyzed separately, including its disconnected contribution. More details are provided in appendix D.
} 
4. In addition, the correlator $G^{\rho \rho}\left(x_{0}\right)$ can be calculated in infinite volume beyond $x_{0}^{\text {cut }}$ via eq. (4.10), and from there $a_{\mu}^{\text {hvp }}$ is obtained. This estimator corrects for the dominant finite-size effects.

A discussion of the systematic error associated with the procedure is presented in appendix C. In steps 3 and 4 , the lattice data $G^{f}\left(x_{0}\right)_{\text {inter }}$ is used directly up to $x_{0}^{\text {cut }}$. We remark that the parameters describing the pion form factor must, in general, be determined simultaneously from the spectrum and finite-volume matrix elements; however in the present case we exploit the fact that the lowest two energy levels are only weakly dependent on $\Gamma_{\rho}$.

To determine the GS $\rho$-mass from the smeared-smeared correlator (step 1) we have proceeded in the following way. For ensembles O7, N6, F7, F6, B6 and A5, the $\rho$-mass parameter of the GS model was extracted from a single-exponential fit to the smearedsmeared correlator. We have checked in these cases that, if the form factor is described by the GS model, identifying the lowest-lying energy-level with the GS $\rho$-mass is an excellent approximation, almost irrespectively of the value of $\Gamma_{\rho}$. On ensemble G8, we have applied a two-exponential fit where the first energy level is set to $E_{1}=2 \sqrt{m_{\pi}^{2}+(2 \pi / L)^{2}}$ by hand and the second exponential is fitted and its mass identified with $m_{\rho}$. In addition, both amplitudes $A_{1}$ and $A_{2}$ are fitted. Even with this three-parameter fit, we encountered a few bootstrap samples where the fit was unstable. Therefore we stabilized the fit in the following way: based on the ensembles with $m_{\pi}<400 \mathrm{MeV}$, we performed an extrapolation of the GS $\rho$-mass linearly in $m_{\pi}^{2}$ to the pion mass of the G8 ensemble, resulting in $m_{\rho}^{\text {xtrap }}=$ $(797 \pm 15) \mathrm{MeV}$. We then used this information as a Bayesian prior, adding $\Delta \chi^{2}=\left(m_{\rho}-\right.$ $\left.m_{\rho}^{\text {xtrap }}\right)^{2} / \sigma^{2}$ to the $\chi^{2}$, where $\sigma$ was varied between 15 and $120 \mathrm{MeV}$. We found that the fit result was stable as long as $\sigma \leq 60 \mathrm{MeV}$.

Figure 2 shows the effect of describing the long-distance part of the correlator using the GS model as compared to using a single exponential for ensembles O7 and G8. Both the finite-volume and the infinite-volume versions are displayed. While the differences do not seem very dramatic, their impact on $a_{\mu}^{\text {hvp }}$ is significant, particularly because the effect of the two-pion continuum increases as the chiral limit is approached. By inserting the GSbased extensions of the iso-vector correlator $G^{\rho \rho}$ for $x_{0}>x_{0}^{\text {cut }}$ in finite and infinite volume into eq. (4.9) one can compute the corresponding estimates of $\left(a_{\mu}^{\mathrm{hvp}}\right)^{u d}$. The results are summarized in table 5 .

\subsection{Comparison of $a_{\mu}^{\text {hvp }}$}

We are now in a position to compare the estimates for $a_{\mu}^{\text {hvp }}$ obtained from different procedures described in the previous subsections. Obviously, this comparison refers only to the data without finite-volume corrections, since the latter have only been quantified for the TMR. The results listed in tables 3,4 and 5 show certain trends regarding their statistical errors. For instance, all three methods yield comparable statistical accuracy for the strange quark contribution $\left(a_{\mu}^{\text {hvp }}\right)^{s}$. The light quark contribution $\left(a_{\mu}^{\text {hvp }}\right)^{u d}$ is equally precise when determined via the TMR or via Padé $[1,1]$ fits below $Q_{\text {cut }}^{2}$. By contrast, constraining the low- $Q^{2}$ behaviour via time moments yields much smaller errors for $\left(a_{\mu}^{\text {hvp }}\right)^{u d}$. Finally, 
the TMR is statistically by far the most precise method for determining the charm quark contribution $\left(a_{\mu}^{\text {hvp }}\right)^{c}$.

One might expect the results obtained using all three variants to agree for each individual ensemble. However, it is easy to see from tables 3-5 that this is not always the case. The largest differences, which amount to about 10\%, are observed for the charm quark contribution. By contrast, one mostly finds agreement among the estimates for $\left(a_{\mu}^{\text {hvp }}\right)^{u d}$ at the level of one or two standard deviations. Another interesting observation is the fact that the differences among estimates determined via the three methods decrease at smaller lattice spacing. Thus, the spread of results among individual ensembles can be attributed to a large part to the presence of lattice artefacts. This interpretation is further supported by the observation - discussed in the next section - that the estimates for $a_{\mu}^{\text {hvp }}$ at the physical point agree within the quoted uncertainties.

\section{Chiral and continuum extrapolations}

We now describe our procedure for determining $a_{\mu}^{\text {hvp }}$ at the physical point, i.e. for vanishing lattice spacing and at the physical pion mass. We start by noting that there is no theoretically preferred ansatz which describes the chiral behaviour of $a_{\mu}^{\text {hvp }}$ in the range of pion masses which is usually considered in lattice simulations. We have therefore subjected the sets of results listed in tables 3,4 and 5 to simultaneous chiral and continuum extrapolations, using a variety of functional forms that parameterize the dependence on the pion mass and the lattice spacing, i.e.

$$
\begin{array}{ll}
\text { Fit A: } & \alpha_{1}+\alpha_{2} m_{\pi}^{2}+\alpha_{3} m_{\pi}^{2} \ln m_{\pi}^{2}+\alpha_{4} a, \\
\text { Fit B: } & \beta_{1}+\beta_{2} m_{\pi}^{2}+\beta_{3} m_{\pi}^{4}+\beta_{4} a, \\
\text { Fit C: } & \gamma_{1}+\gamma_{2} m_{\pi}^{2}+\gamma_{3} a \\
\text { Fit D: } & \delta_{1}+\delta_{2} a
\end{array}
$$

with fit parameters $\alpha_{1}, \alpha_{2}, \ldots, \delta_{2}$. All four ansätze contain a term of order $a$, since the operators whose matrix elements determine the vacuum polarization are not fully $\mathrm{O}(a)$ improved. The terms proportional to $m_{\pi}^{2} \ln m_{\pi}^{2}$ and $m_{\pi}^{4}$ in fits $\mathrm{A}$ and $\mathrm{B}$, respectively, account for the curvature in the chiral behaviour of the light-quark contribution $\left(a_{\mu}^{\mathrm{hvp}}\right)^{u d}$. By contrast, the pion mass dependence of $\left(a_{\mu}^{\mathrm{hvp}}\right)^{s}$ and $\left(a_{\mu}^{\mathrm{hvp}}\right)^{c}$ is mostly linear or even constant, which motivates the absence of such terms in fits $\mathrm{C}$ and $\mathrm{D}$.

In order to estimate systematic errors associated with variations of our fitting and analysis procedures we have employed the so-called "extended frequentist's method" (EFM) [82, 83]. When combined with the bootstrap method designed for the estimation of statistical errors one obtains the fit result from the median of the joint distribution, while statistical and systematic errors are represented by the lower and upper bounds of the central $68 \%$. An overview of all fitting and analysis variants which enter the EFM are presented in table 6 . As regards variations of the ansatz for the chiral fit, we note that two additional functional forms were discussed in ref. [93], namely a fit including one inverse power of $m_{\pi}^{2}$, as well as a ChPT-inspired function containing a term proportional to $\ln m_{\pi}^{2}$ (i.e. without 


\begin{tabular}{|c|c|c|c|}
\hline Hybrid Method & light & strange & charm \\
\hline Fit ansatz & $\mathrm{A}, \mathrm{B}$ & $\mathrm{A}, \mathrm{B}, \mathrm{C}$ & $\mathrm{C}, \mathrm{D}$ \\
\hline $\begin{array}{l}\text { Cuts in } m_{\pi} \\
\quad \text { and } a\end{array}$ & $\begin{array}{c}\text { no cuts } \\
\text { cut } 1^{*} \\
\text { cut } 2^{\dagger} \\
\text { cuts } 1 \text { and } 2\end{array}$ & $\begin{array}{c}\text { no cuts } \\
\text { cut } 1 \\
\text { cut } 2 \\
\text { cuts } 1 \text { and } 2\end{array}$ & $\begin{array}{c}\text { no cuts } \\
\text { cut } 1 \\
\text { cut } 2 \\
\text { cuts } 1 \text { and } 2\end{array}$ \\
\hline IR regime & $\begin{array}{l}Q_{\text {cut }}^{2} \approx 0.5 \mathrm{GeV}^{2} \\
Q_{\text {cut }}^{2}<0.5 \mathrm{GeV}^{2}\end{array}$ & $\begin{array}{l}Q_{\text {cut }}^{2} \approx 0.5 \mathrm{GeV}^{2} \\
Q_{\text {cut }}^{2}<0.5 \mathrm{GeV}^{2}\end{array}$ & $\begin{array}{l}\text { Polynomial } \\
\text { Padé }\end{array}$ \\
\hline $\begin{array}{c}\text { Current } \\
\text { renormalization }\end{array}$ & $Z_{\mathrm{V}}^{\left(m_{u d}\right)}$ & $Z_{\mathrm{V}}^{\left(m_{s}\right)}$ & $\begin{array}{c}Z_{\mathrm{V}}^{\left(m_{c}\right)} \\
Z_{\mathrm{V}}\left(1+b_{\mathrm{V}} a m_{c}\right)\end{array}$ \\
\hline TMR & light & strange & charm \\
\hline Fit ansatz & $\mathrm{A}, \mathrm{B}$ & $\mathrm{A}, \mathrm{B}, \mathrm{C}$ & $\mathrm{C}, \mathrm{D}$ \\
\hline $\begin{array}{l}\text { Cuts in } m_{\pi} \\
\quad \text { and } a\end{array}$ & $\begin{array}{c}\text { no cuts } \\
\text { cut } 1 \\
\text { cut } 2 \\
\text { cuts } 1 \text { and } 2\end{array}$ & $\begin{array}{c}\text { cut } 2 \\
\text { cuts } 1 \text { and } 2\end{array}$ & $\begin{array}{c}\text { cut } 2 \\
\text { cuts } 1 \text { and } 2\end{array}$ \\
\hline IR regime & $\begin{array}{l}\text { single exponential }{ }^{\ddagger} \\
\text { Gounaris-Sakurai }\end{array}$ & single exponential & single exponential \\
\hline $\begin{array}{c}\text { Current } \\
\text { renormalization }\end{array}$ & $Z_{\mathrm{V}}^{\left(m_{u d}\right)}$ & $Z_{\mathrm{V}}^{\left(m_{s}\right)}$ & $\begin{array}{c}Z_{\mathrm{V}}^{\left(m_{c}\right)} \\
Z_{\mathrm{V}}\left(1+b_{\mathrm{V}} a m_{c}\right)\end{array}$ \\
\hline
\end{tabular}

${ }^{*}$ Cut 1: $m_{\pi}<400 \mathrm{MeV}$

${ }^{\dagger}$ Cut 2: $a<0.07 \mathrm{fm}$

$\ddagger$ Single exponential is not used as a variation with the GS model including the FV correction

Table 6. Overview of variants of the fitting and analysis procedures which enter the estimation of systematic errors via the extended frequentist method. We focus on the hybrid method with the low- $Q^{2}$ behaviour determined by fits, as well as the TMR. The meaning of the various cuts is explained below the table.

the factor of $m_{\pi}^{2}$ multiplying the logarithm). We note that an ansatz containing $\ln m_{\pi}^{2}$ has a compelling justification only for $m_{\pi}<m_{\mu}$ [93] and does not apply to the situation realized in our simulations. While an inverse power of $m_{\pi}^{2}$ does arise in the slope of $\Pi\left(Q^{2}\right)$ at $Q^{2}=0$ via the numerically subdominant pion loop contribution [50], it may over-amplify the dependence of $a_{\mu}^{\text {hvp }}$ on $m_{\pi}^{2}$ near the physical pion mass [93]. We have therefore excluded terms like $1 / m_{\pi}^{2}$ and $\ln m_{\pi}^{2}$ from our EFM analysis. As a further check we have performed tentative fits based on a modified version of fit A, in which $\alpha_{3} m_{\pi}^{2} \ln m_{\pi}^{2}$ was replaced by $\alpha_{3} \ln m_{\pi}^{2}$. The resulting estimates for $a_{\mu}^{\text {hvp }}$ at the physical point are well within the total error obtained by the EFM procedure. Thus, we conclude that the uncertainty associated with the chiral extrapolation has been quantified reliably.

The systematics of the chiral and continuum extrapolation can be investigated by varying the fit ansatz and by imposing different cuts in the maximum pion mass and the 


\begin{tabular}{|cccc|}
\hline & Hybrid Method & TMR & TMR + FV \\
\hline$\left(a_{\mu}^{\text {hvp }}\right)^{u d}$ & $556.6 \pm 25.3 \pm 16.9$ & $551.3 \pm 24.7 \pm 28.9$ & $588.2 \pm 31.7 \pm 16.6$ \\
$\left(a_{\mu}^{\text {hvp }}\right)^{s}$ & $51.9 \pm 2.1 \pm 1.7$ & $51.1 \pm 1.7 \pm 0.4$ & $51.1 \pm 1.7 \pm 0.4$ \\
$\left(a_{\mu}^{\text {hvp }}\right)^{c}$ & $13.9 \pm 0.8 \pm 0.9$ & $14.3 \pm 0.2 \pm 0.1$ & $14.3 \pm 0.2 \pm 0.1$ \\
\hline$\left(a_{\mu}^{\text {hvp }}\right)^{u d s c}$ & $623.1 \pm 25.4 \pm 19.7$ & $616.7 \pm 24.8 \pm 28.9$ & $653.6 \pm 31.8 \pm 16.6$ \\
\hline
\end{tabular}

Table 7. Summary of results for the hadronic vacuum polarization contribution (in units of $10^{-10}$ ) at the physical point. The first error is statistical while the second denotes the systematic uncertainty as estimated via the variations listed in table 6 . The rightmost column contains the estimate for $\left(a_{\mu}^{\text {hvp }}\right)^{u d}$ including corrections for finite-size effects.

lattice spacing $a$. Another important systematic effect is associated with constraining the deep infrared regime of the vacuum polarization: in the case of the hybrid method we have used different values of the momentum scale $Q_{\text {cut }}^{2}$ below which the vacuum polarization function is described by a low-order Padé approximant.

For the TMR we have included two different variants for extending the vector correlator $G^{u d}\left(x_{0}\right)$ beyond $x_{0}^{\text {cut }}$, the first being the single-exponential ansatz, with the GS model (excluding the finite-volume correction) as an alternative. The GS-parameterization including the finite-volume shift was extrapolated separately. In this case we did not study effects of another ansatz for describing the infrared behaviour. For the strange and charm quark contributions we only used the single-exponential extension, since the estimates for $\left(a_{\mu}^{\text {hvp }}\right)^{s}$ and $\left(a_{\mu}^{\mathrm{hvp}}\right)^{c}$ do not depend strongly on the details of the corresponding vector correlators for $x_{0} \gtrsim 1.2 \mathrm{fm}$.

The contribution from the charm quark to $a_{\mu}^{\text {hvp }}$ is particularly sensitive to the discretization and renormalization effects. This can be inferred already from the fact that the estimates for $\left(a_{\mu}^{\mathrm{hvp}}\right)^{c}$ differ by $30-40 \%$ between our coarsest and finest lattice spacing (see tables 3-5). Furthermore, combined chiral and continuum fits of the data including all three lattice spacings produce large values of $\chi^{2} /$ dof, which is particularly pronounced for the data obtained using the TMR. We have therefore consistently excluded the TMR-data for $\left(a_{\mu}^{\text {hvp }}\right)^{c}$ computed at the coarsest lattice spacing from the extrapolations to the physical point. Furthermore, in order to study whether the details of fixing the renormalization factor of the local vector current have a noticeable systematic effect on the extrapolation we have repeated the fits of $\left(a_{\mu}^{\mathrm{hvp}}\right)^{c}$ using the factor $Z_{\mathrm{V}}\left(1+b_{\mathrm{V}} a m_{f}\right)$ instead of $Z_{\mathrm{V}}^{\left(m_{f}\right)}$.

Another comment on the use of time moments to constrain the low- $Q^{2}$ dependence of $\Pi\left(Q^{2}\right)$ is in order. We found that the combined fits to the results listed in table 4 produced values of $\chi^{2} /$ dof between 5 and 10 , regardless of the fit ansatz or of any other procedural variation. The most likely explanation is the smallness of the statistical errors relative to the intrinsic fluctuations in the chiral and continuum behaviour among the ensembles. Therefore we will focus on the TMR and the Hybrid method as implemented via Padé fits in the following. 

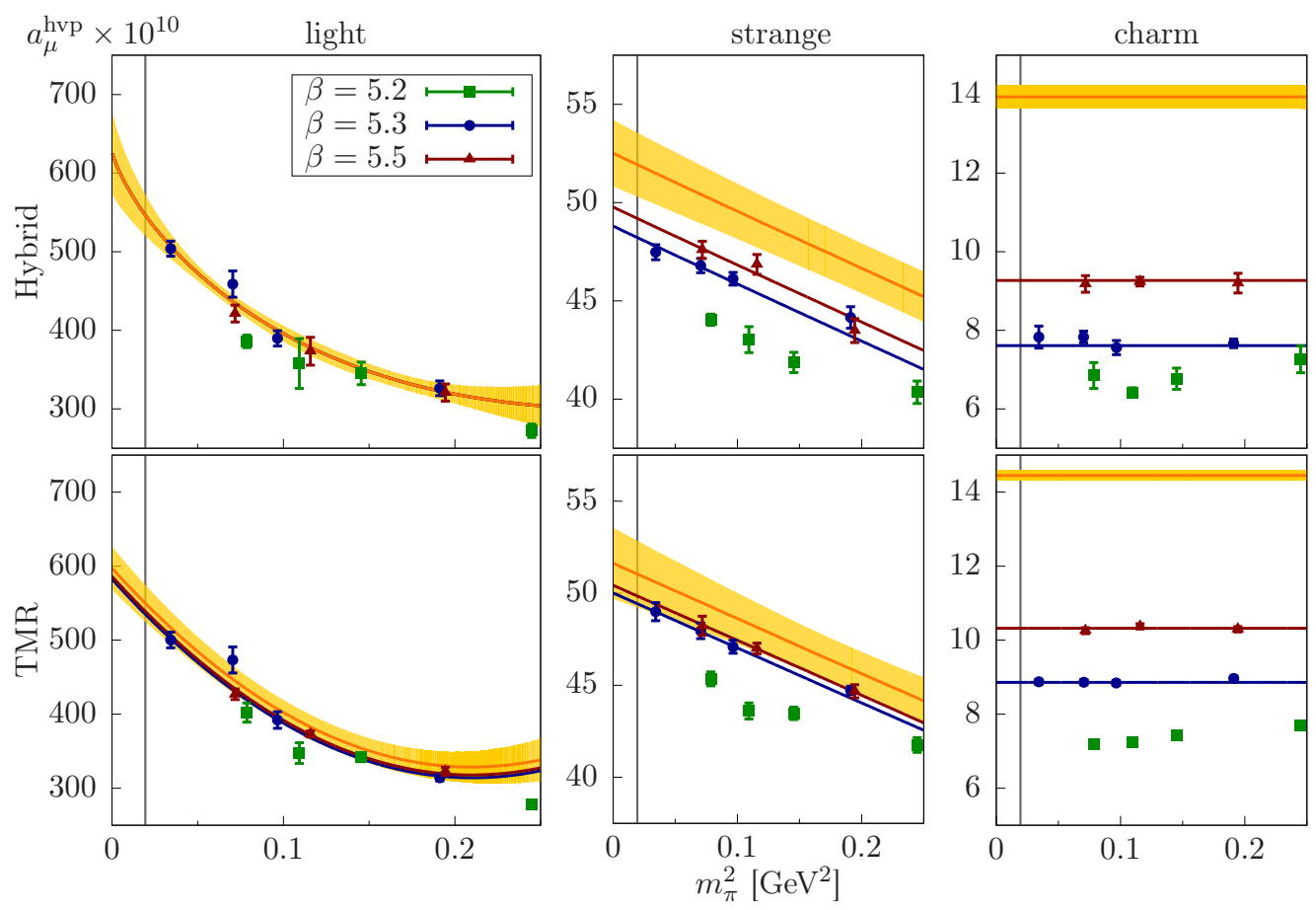

Figure 3. Examples of chiral and continuum extrapolations of the light, strange and charm quark contributions to $a_{\mu}^{\text {hvp }}$ for the hybrid (above) and TMR (below) methods. Yellow bands correspond to the chiral behaviour in the continuum limit, while the dark red and blue curves represent the pion mass dependence at $\beta=5.5$ and 5.3. The physical value of the pion mass is indicated by the vertical lines.
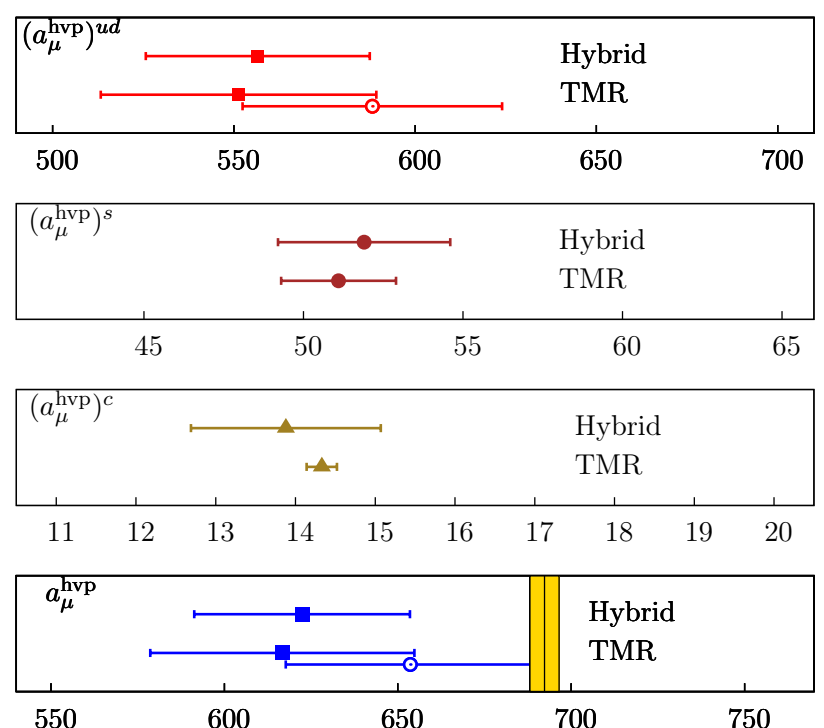

Figure 4. Comparison of results for the different flavour contributions to $a_{\mu}^{\text {hvp }}$ in units of $10^{-10}$. Open circles denote the results based on the finite-volume corrected estimates of the light quark contribution. The yellow vertical band denotes the result obtained from dispersion theory [3]. 
Examples of our chiral and continuum extrapolations are shown in figure 3 while table 7 contains an overview of results for the individual flavour contributions to $a_{\mu}^{\text {hvp }}$ at the physical point. We observe good agreement between the Hybrid and TMR methods. We also note that the inclusion of the finite-volume correction via the GS model produces a sizeable upward shift in $\left(a_{\mu}^{\mathrm{hvp}}\right)^{u d}$. This is also apparent from figure 4 .

There are two additional sources of systematic error which we discuss separately. The first concerns the impact of the uncertainty in the lattice scale: in order to make contact between the kernel function $K\left(Q^{2} ; m_{\mu}^{2}\right)$ and the VPF $\hat{\Pi}\left(Q^{2}\right)$ computed on the lattice one must express the dimensionless momentum scale $(a Q)$ in units of the muon mass. In our calculation the lattice spacing is known with a precision at the level of $1 \%$ (see table 1 ). To assess the systematic error associated with scale setting we have repeated the chiral and continuum fits for the Hybrid method, using the upper and lower values of $a$ as defined by the $1-\sigma$ bands. The variation of the lattice scale by $\pm 1 \%$ increased the overall systematic error in $\left(a_{\mu}^{\text {hvp }}\right)^{u d}$ as estimated via the EFM by $1.8 \%$. Given the ultimate precision goal of less than $1 \%$ uncertainty, this is a rather large systematic effect. For the TMR we have derived an entirely consistent estimate of the scale setting uncertainty using the representation of the kernel function $\widetilde{K}\left(x_{0} ; m_{\mu}\right)$. Details are presented in appendix B.2.

The second additional uncertainty is associated with the contributions from disconnected diagrams. In appendix D we present our calculation of quark-disconnected contributions on a subset of our ensembles (E5 and F6). The main result of that investigation is the derivation of a conservative upper bound on the magnitude of the disconnected contribution. Our findings indicate that quark-disconnected diagrams decrease the estimate of $a_{\mu}^{\text {hvp }}$ by at most $2 \%$.

As our final estimate for the hadronic vacuum polarization contribution we quote the result from the TMR including the finite-volume corrections based on the GS-parameterization. Adding the contributions from the light, strange and charm quarks we arrive at

$$
a_{\mu}^{\text {hvp }}=\left(654 \pm 32_{\text {stat }} \pm 17_{\text {syst }} \pm 10_{\text {scale }} \pm 7_{\mathrm{FV}_{-10}^{+0} \text { disc }}^{+0}\right) \cdot 10^{-10} .
$$

The quoted systematic error was estimated via the EFM considering the variations listed in the lower part of table 6 . The scale uncertainty (third error) amounts to the increase in the systematic error estimate when the lattice spacing is shifted by $\pm 1 \sigma$ and the corresponding variations are included in the EFM procedure for the Hybrid method. As described in appendix C, we assign an uncertainty of $20 \%$ to the determination of the finite-volume shift in $\left(a_{\mu}^{\text {hvp }}\right)^{u d}$. This produces an additional systematic error of $\pm 7 \cdot 10^{-10}$. Finally, we estimate that quark-disconnected diagrams reduce the value of $a_{\mu}^{\text {hvp }}$ by at most $10 \cdot 10^{-10}$ when the latter is computed using connected correlators only.

Our calculation has been performed in two-flavour QCD, and hence our results will be affected by the quenching of the strange and, to a lesser extent, the charm quark. Since we know of no reliable way of estimating the associated systematic effect, we leave it unspecified and caution the reader that this has to be taken into account when comparing our result to phenomenology or other lattice determinations. We add that our results are in good agreement with those of refs. [33, 39] which were performed for $N_{\mathrm{f}}=2+1+1$ flavours. 


\section{Conclusions}

We have presented a lattice calculation of the hadronic vacuum polarization contribution to the muon $g-2$ addressing all sources of systematic error, except isospin breaking and the effects of dynamical strange and charm quarks. Given the overall uncertainty of $6 \%$ it is unlikely that our result, presented in eq. (5.5), is strongly biased by the omission of these effects. Our estimate is lower than the current value from dispersion theory but in agreement within the error of our calculation. Lattice determinations of $a_{\mu}^{\text {hvp }}$ have become more accurate in recent years, yet the target precision of $\lesssim 1 \%$ has not been reached so far. While the statistical accuracy can be straightforwardly improved by an increased numerical effort, this is a lot more difficult for some of the various sources of systematics error.

In this paper we have investigated several complementary methods designed to control the infrared regime. One important lesson is the observation that this issue is strongly linked with the question of finite-volume effects. Our investigation of the long-distance regime of the vector correlator by means of the Gounaris-Sakurai parameterization of the pion form factor revealed that finite-volume effects are significant. They amount to a $5 \%$ shift in the value of $a_{\mu}^{\text {hvp }}$ for $m_{\pi} L \approx 4$ and near-physical pion masses. While this is consistent with similar estimates based on effective field theories (see, for instance, refs. [39, $40,78,94]$ ), a direct calculation, performed at sufficiently large $m_{\pi} L$, which demonstrates that finite-volume effects are under control is still lacking. Based on the Gounaris-Sakurai model, we estimate that finite-volume effects are below the percent level when $m_{\pi} L \gtrsim 6$. Another important issue is the individual contribution from the charm quark, $\left(a_{\mu}^{\text {hvp }}\right)^{c}$, which amounts to about $2 \%$ of the total value. Given that $\left(a_{\mu}^{\mathrm{hvp}}\right)^{c}$ is quite sensitive to lattice artefacts, it is of vital importance to reliably control the continuum limit if one aims at subpercent precision. Furthermore, scale setting has a large influence on the overall accuracy. Our analysis has shown that an extremely precise calibration of the lattice spacing significantly below the percent level - is indispensable for a lattice determination of $a_{\mu}^{\text {hvp }}$ that is competitive with the dispersive approach.

\section{Acknowledgments}

The authors are indebted to Jeremy Green for the calculation of renormalization factors. We are grateful to our colleagues within the CLS initiative for sharing ensembles. Our calculations were partly performed on the HPC Clusters "Wilson" and "Clover" at the Institute for Nuclear Physics, University of Mainz. We thank Dalibor Djukanovic and Christian Seiwerth for technical support. We are grateful for computer time allocated to project HMZ21 on the BG/Q "JUQUEEN" computer at NIC, Jülich. This work was granted access to the HPC resources of the Gauss Center for Supercomputing at Forschungszentrum Jülich, Germany, made available within the Distributed European Computing Initiative by the PRACE-2IP, receiving funding from the European Community's Seventh Framework Programme (FP7/2007-2013) under grant agreement RI-283493. This work was supported by the DFG through SFB 1044, and by the Rhineland-Palatinate Research Initiative. MDM was partially supported by the Danish National Research Foundation under grant number 
DNRF:90. G.H. acknowledges support by the Spanish MINECO through the Ramón y Cajal Programme and through the project FPA2015-68541-P and by the Centro de excelencia Severo Ochoa Program SEV-2012-0249. V.G. acknowledges support from UK Consolidated Grant ST/L000296/1.

\section{A Renormalization of the vector current}

Here we describe the procedure used to determine the (mass-dependent) renormalization factor of the vector current from the quark-connected contribution to the three-point function

$$
C_{3}\left(t, t_{s}\right)=\sum_{\vec{x}, \vec{y}}\left\langle O\left(\vec{x}, t_{s}\right) V_{0, f}^{\mathrm{loc}}(\vec{y}, t) O^{\dagger}(\overrightarrow{0}, 0)\right\rangle,
$$

and the two-point function

$$
C_{2}(t)=\sum_{\vec{x}}\left\langle O(\vec{x}, t) O^{\dagger}(\overrightarrow{0}, 0)\right\rangle
$$

where the operator $O$ is given by $O=\bar{\psi}_{f^{\prime}} \gamma_{5} \psi_{f}$, and $V_{\mu, f}^{\text {loc }}$ is defined in eq. (3.2). Choosing the source-sink separation $t_{s}$ as $t_{s}=T / 2$ one can form the ratio

$$
R(t, T / 2) \equiv \frac{C_{3}(t, T / 2)}{C_{2}(T / 2)},
$$

as well as the difference

$$
d(t) \equiv R(t, T / 2)-R(t+T / 2, T / 2) .
$$

By fitting $d(t)$ to a constant $Q_{\mathrm{V}}$ over a Euclidean time interval one can determine the renormalization factor $Z_{\mathrm{V}}^{\left(m_{f}\right)}$ by imposing

$$
Z_{\mathrm{V}}^{\left(m_{f}\right)} Q_{\mathrm{V}}=1 .
$$

Table 8 shows a compilation of results for $Z_{\mathrm{V}}^{\left(m_{f}\right)}$ computed on all ensemble used in this study.

The renormalization condition of eq. (A.5) depends on the flavour $f^{\prime}$ of the spectator quark. On ensemble E5 we have studied all possible combinations of $f$ and $f^{\prime}$ (i.e. $u d, s$ and $c$ ). Our findings indicate that spectator quark effects are below $1 \%$, with the strongest influence seen in the case of the renormalization of the charm quark contribution to the vector current.

\section{B The QED kernel in the time-momentum representation}

The vector correlator in the time-momentum representation is given in eq. (2.6). The master equation to compute $a_{\mu}^{\text {hvp }}$ from it is [51]

$$
\begin{aligned}
a_{\mu}^{\mathrm{hvp}} & =\left(\frac{\alpha}{\pi}\right)^{2} \int_{0}^{\infty} d t G(t) \widetilde{K}\left(t ; m_{\mu}\right), \\
\widetilde{K}\left(t ; m_{\mu}\right) & \equiv \tilde{f}(t)=8 \pi^{2} \int_{0}^{\infty} \frac{d \omega}{\omega} f\left(\omega^{2}\right)\left[\omega^{2} t^{2}-4 \sin ^{2}\left(\frac{\omega t}{2}\right)\right],
\end{aligned}
$$




\begin{tabular}{|cccc|}
\hline Run & $Z_{\mathrm{V}}^{\left(m_{u d}\right)}$ & $Z_{\mathrm{V}}^{\left(m_{s}\right)}$ & $Z_{\mathrm{V}}^{\left(m_{c}\right)}$ \\
\hline A3 & $0.73228(29)$ & $0.74625(30)$ & $1.08944(62)$ \\
A4 & $0.72924(42)$ & $0.74773(20)$ & $1.09915(32)$ \\
A5 & $0.72724(43)$ & $0.74803(21)$ & $1.10167(68)$ \\
B6 & $0.72646(44)$ & $0.74869(17)$ & $1.10525(29)$ \\
\hline E5 & $0.74418(33)$ & $0.75829(22)$ & $1.04630(43)$ \\
F6 & $0.74143(14)$ & $0.75924(08)$ & $1.04948(35)$ \\
F7 & $0.74011(23)$ & $0.75950(12)$ & $1.04968(30)$ \\
G8 & $0.73887(10)$ & $0.75983(13)$ & $1.05043(27)$ \\
\hline N5 & $0.76524(07)$ & $0.77513(08)$ & $0.96698(16)$ \\
N6 & $0.76315(17)$ & $0.77548(07)$ & $0.96663(17)$ \\
O7 & $0.76193(14)$ & $0.77562(08)$ & $0.96749(16)$ \\
\hline
\end{tabular}

Table 8. Results for the mass-dependent renormalization factor $Z_{\mathrm{V}}^{\left(m_{f}\right)}$ defined in eq. (A.5), computed for degenerate active and spectator quarks, $f=f^{\prime}=u d, s, c$. Numbers in parentheses denote statistical errors.

with the momentum-space kernel given by ${ }^{3}$

$$
\begin{aligned}
K\left(s ; m_{\mu}^{2}\right) & \equiv f(s)=\frac{1}{m_{\mu}^{2}} \cdot \hat{s} \cdot Z(\hat{s})^{3} \cdot \frac{1-\hat{s} Z(\hat{s})}{1+\hat{s} Z(\hat{s})^{2}}, \\
Z(\hat{s}) & =-\frac{\hat{s}-\sqrt{\hat{s}^{2}+4 \hat{s}}}{2 \hat{s}}, \quad \hat{s}=\frac{s}{m_{\mu}^{2}} .
\end{aligned}
$$

\section{B.1 Derivation of a representation of the kernel function}

Our goal is to obtain a simple and accurate representation of $\tilde{f}(t)$ which can be used straightforwardly in the expression for $a_{\mu}^{\text {hvp }}$ via eq. (B.1). Since $\tilde{f}(t)$ has units of $\mathrm{GeV}^{-2}$ and only involves the muon mass as an external scale, it is clear that $m_{\mu}^{2} \tilde{f}(t)$ must be a dimensionless function in the variable $\left(m_{\mu} t\right)$.

For the following derivation it is convenient to set the muon mass to unity and restore the units by dimensional analysis at the end of the calculation. The function $f\left(\omega^{2}\right)$ can be simplified $(\omega>0)$,

$$
f\left(\omega^{2}\right)=\frac{1}{\omega \sqrt{\omega^{2}+4}}-1+\frac{\omega}{2}\left(\sqrt{\omega^{2}+4}-\omega\right)
$$

and hence $f\left(\omega^{2}\right) / \omega$ goes like $1 / \omega^{2}$ at $\omega=0$.

The key observation is that $\tilde{f}(t)$ can be expressed in terms of the auxiliary function

$$
\tilde{g}_{\epsilon}(t)=\int_{0}^{\infty} \frac{d \omega}{\sqrt{\omega^{2}+\epsilon^{2}}} f\left(\omega^{2}+\epsilon^{2}\right) \cos (\omega t)
$$

\footnotetext{
${ }^{3}$ Our kernel $K$ matches the function $f$ introduced in [28].
} 
as

$$
\tilde{f}(t)=16 \pi^{2} \lim _{\epsilon \rightarrow 0}\left(\tilde{g}_{\epsilon}(t)-\left(\tilde{g}_{\epsilon}(0)+\tilde{g}_{\epsilon}^{\prime}(0) t+\frac{1}{2} \tilde{g}_{\epsilon}^{\prime \prime}(0) t^{2}\right)\right)
$$

Note that $\epsilon>0$ serves as an infrared regulator which is removed at the end of the calculation. In fact, we note that the regulation is only necessary for the first two terms in $f\left(\omega^{2}\right)$. One finds that the contribution of the second and third term in eq. (B.5) to $\tilde{g}_{\epsilon}(t)$ can be expressed in terms of modified Bessel functions, $K_{0}$ and $K_{1}$. The first term in eq. (B.5) is the most complicated: it involves the evaluation of the integral

$$
I_{\epsilon}(t)=\int_{0}^{\infty} \frac{d \omega}{\omega^{2}+\epsilon^{2}} \frac{\cos (\omega t)}{\sqrt{\omega^{2}+4}}
$$

which satisfies

$$
I_{\epsilon}^{\prime \prime}(t)-\epsilon^{2} I_{\epsilon}(t)=-K_{0}(2 t), \quad I_{\epsilon}(0)=\frac{\pi}{4 \epsilon}-\frac{1}{4}+\mathrm{O}(\epsilon), \quad I_{\epsilon}^{\prime}(0)=0 .
$$

The two linearly independent solutions of the homogeneous equation are $e^{ \pm \epsilon t}$. A particular solution $I_{p}(t)$ of the inhomogeneous equation can be found using the standard integral representation

$$
K_{0}(t)=\int_{1}^{\infty} d u \frac{e^{-t u}}{\sqrt{u^{2}-1}}
$$

and the Laplace transform $I_{p}(t)=\int_{0}^{\infty} d u e^{-u t} \tilde{I}_{p}(u)$, which yields $\tilde{I}_{p}(u)=-\frac{\theta(u-2)}{\left(u^{2}-\epsilon^{2}\right) \sqrt{u^{2}-4}}$. Realizing that $\epsilon$ can be set to zero, we arrive at the representation

$$
I_{p}(t)=-\int_{2}^{\infty} \frac{d u e^{-u t}}{u^{2} \sqrt{u^{2}-4}}=-\int_{0}^{\infty} d v \frac{e^{-t \sqrt{v^{2}+4}}}{\left(v^{2}+4\right)^{3 / 2}}
$$

Noting that $I_{p}(0)=-1 / 4$ and $I_{p}^{\prime}(0)=\pi / 4$, we impose the initial conditions and obtain the full solution up to terms of $\mathrm{O}(\epsilon)$, i.e.

$$
I_{\epsilon}(t)=\frac{\pi}{4}\left(\frac{1}{\epsilon}-t\right)+I_{p}(t)+\mathrm{O}(\epsilon)
$$

The integral $I_{p}(t)$ can be expressed in terms of Meijer's G function [95]. In Mathematica [96], it can be evaluated by a built-in function

$$
I_{p}(t)=\frac{\pi t}{4}+\frac{1}{8} \operatorname{MeijerG}\left[\{\{3 / 2\},\{\}\},\{\{0,1\},\{1 / 2\}\}, t^{2}\right] .
$$

Putting everything together, we have

$$
\tilde{g}_{\epsilon}(t)=\frac{\pi}{4}\left(\frac{1}{\epsilon}-t\right)+I_{p}(t)-K_{0}(\epsilon t)+\frac{1}{2 t^{2}}\left(-2 t K_{1}(2 t)+1\right)+\mathrm{O}(\epsilon) .
$$

From here one obtains straightforwardly, now restoring the units,

$$
\tilde{f}(t)=\frac{2 \pi^{2}}{m_{\mu}^{2}}\left(-2+8 \gamma_{\mathrm{E}}+\frac{4}{\hat{t}^{2}}-2 \pi \hat{t}+\hat{t}^{2}-\frac{8}{\hat{t}} K_{1}(2 \hat{t})+8 \ln (\hat{t})+8 I_{p}(\hat{t})\right), \quad \hat{t}=m_{\mu} t
$$


where $\gamma_{\mathrm{E}}=0.57721566490153286061 \ldots$ is Euler's constant. The expansion of $\tilde{f}(t)$ around the origin yields

$$
\begin{aligned}
m_{\mu}^{2} \tilde{f}(t)= & \frac{\pi^{2} \hat{t}^{4}}{9}+\frac{\pi^{2} \hat{t}^{6}\left(120 \ln (\hat{t})+120 \gamma_{\mathrm{E}}-169\right)}{5400} \\
& +\frac{\pi^{2} \hat{t}^{8}\left(210 \ln (\hat{t})+210 \gamma_{\mathrm{E}}-401\right)}{88200}+\frac{\pi^{2} \hat{t}^{10}\left(360 \ln (\hat{t})+360 \gamma_{\mathrm{E}}-787\right)}{2916000} \\
& +\frac{\pi^{2} \hat{t}^{12}\left(3080 \ln (\hat{t})+3080 \gamma_{\mathrm{E}}-7353\right)}{768398400}+\mathrm{O}\left(\hat{t}^{14}\right)
\end{aligned}
$$

Note that $\tilde{f}(t)$ is not analytic at the origin, due to the appearance of terms proportional to $\ln (\hat{t})$ beyond fourth order. The expansion at large $t$ yields

$$
m_{\mu}^{2} \tilde{f}(t)=2 \pi^{2} \hat{t}^{2}-4 \pi^{3} \hat{t}+4 \pi^{2}\left(-1+4 \gamma_{\mathrm{E}}+4 \ln (\hat{t})\right)+\frac{8 \pi^{2}}{\hat{t}^{2}}-\frac{2 \pi^{5 / 2}}{\sqrt{\hat{t}}} e^{-2 \hat{t}}\left(1+\mathrm{O}\left(\hat{t}^{-1}\right)\right) .
$$

For a numerical evaluation, we propose the following. Up to $\hat{t}=1.05$, the expansion of eq. (B.16) around the origin provides an estimate of $\tilde{f}(t)$ with a relative accuracy better than $3.3 \cdot 10^{-6}$. Beyond that point, the series

$$
\begin{aligned}
m_{\mu}^{2} \tilde{f}(t)=2 \pi^{2} \hat{t}^{2}-4 \pi^{3} \hat{t} & +4 \pi^{2}\left(4 \ln (\hat{t})+4 \gamma_{\mathrm{E}}-1\right)+\frac{8 \pi^{2}}{\hat{t}^{2}} \\
-\frac{2 \pi^{5 / 2}}{\sqrt{\hat{t}}} e^{-2 \hat{t}}( & 0.0197159\left(\hat{t}^{-1}-0.7\right)^{6}-0.0284086\left(\hat{t}^{-1}-0.7\right)^{5} \\
& +0.0470604\left(\hat{t}^{-1}-0.7\right)^{4}-0.107632\left(\hat{t}^{-1}-0.7\right)^{3} \\
& \left.+0.688813\left(\hat{t}^{-1}-0.7\right)^{2}+4.71371\left(\hat{t}^{-1}-0.7\right)+3.90388\right)
\end{aligned}
$$

can be used. Its accuracy is also better than $3.3 \cdot 10^{-6}$ for all $\hat{t} \geq 1.05$. Note that the integrand for $a_{\mu}$ is expected to be very small beyond $4 \mathrm{fm}$, corresponding to $\hat{t}>2.14$; see figure 4 in [51].

\section{B.2 Sensitivity of $a_{\mu}^{\text {hvp }}$ to the lattice scale setting}

The representation for the kernel function $\tilde{f}$ derived above can be used to study the sensitivity of $a_{\mu}^{\text {hvp }}$ on the uncertainty in the determination of the lattice scale. Standard error propagation implies that the uncertainty $\Delta \Lambda$ on the observable $\Lambda$ that sets the lattice scale translates into a corresponding uncertainty in $a_{\mu}^{\text {hvp }}$ according to

$$
\Delta a_{\mu}^{\mathrm{hvp}}=\left|\Lambda \frac{d a_{\mu}^{\mathrm{hvp}}}{d \Lambda}\right| \cdot \frac{\Delta \Lambda}{\Lambda}=\left|M_{\mu} \frac{d a_{\mu}^{\mathrm{hvp}}}{d M_{\mu}}\right| \cdot \frac{\Delta \Lambda}{\Lambda},
$$

where $M_{\mu} \equiv m_{\mu} / \Lambda$ denotes the muon mass in units of $\Lambda$. To evaluate the derivative, we note that $t \tilde{f}^{\prime}(t)-\tilde{f}(t)=J(t)$, with

$$
m_{\mu}^{2} J(t) \equiv \frac{2 \pi^{2}}{\hat{t}^{2}}\left(\hat{t}^{4}+\left(10-8 \gamma_{\mathrm{E}}\right) \hat{t}^{2}+4 \hat{t}\left(\left(\hat{t}^{2}+6\right) K_{1}(2 \hat{t})-2 \hat{t} \ln (\hat{t})+4 \hat{t} K_{0}(2 \hat{t})\right)-12\right) .
$$


A short calculation then leads to

$$
M_{\mu} \frac{d a_{\mu}^{\mathrm{hvp}}}{d M_{\mu}}=-a_{\mu}^{\mathrm{hvp}}+\left(\frac{\alpha}{\pi}\right)^{2} \int_{0}^{\infty} d t G(t) J(t) .
$$

As an example application, using the parameterization of the $R$-ratio in [51], which yields $a_{\mu}^{\text {hvp }}=672 \cdot 10^{-10}$, we compute $G\left(x_{0}\right)$ and find $M_{\mu} \frac{d a_{\mu}^{\text {hvp }}}{d M_{\mu}}=1.22 \cdot 10^{-7}$. This means that if the relative scale-setting error $\Delta \Lambda / \Lambda$ is one percent, the impact on the calculation is $\Delta a_{\mu}^{\mathrm{hvp}} / a_{\mu}^{\mathrm{hvp}}=1.8 \%$.

The scale uncertainty $\Delta \Lambda$ also enters via the implicit dependence of $a_{\mu}^{\text {hvp }}$ on dimensionless ratios of quark masses, $m_{u} / \Lambda, m_{d} / \Lambda, m_{s} / \Lambda \ldots$, where the largest effect is expected to come from the light quarks. By studying the chiral behaviour of $a_{\mu}^{\text {hvp }}$ (see figure 3) we have estimated that this produces only a small compensating effect of about $-10 \%$ relative to $M_{\mu} \frac{d a_{\mu}^{\mathrm{hvp}}}{d M_{\mu}}$.

\section{Finite-size effects in the time-momentum representation}

In this appendix we address the finite-size effects on $a_{\mu}^{\text {hvp }}$ in the TMR and our ability to calculate them. Finite-size effects on the time-momentum correlator $G^{\rho \rho}\left(x_{0}\right)$ were computed in [54] based on the Lüscher formalism and the relation between the timelike pion form factor and finite-volume matrix elements [87, 88]. Here we employ exactly the same method and therefore refer the reader to [54] for the relevant technical details. The goal of this appendix is to study the finite-size effects we expect on theoretical grounds at the simulation parameters used in the actual calculation presented in the main text. Several groups have studied finite-size effects on the hadronic vacuum polarization by theoretical means, see [78, 94]. In any comparison, one must keep in mind that the finite-size effects depend on precisely which finite-volume representation of $a_{\mu}^{\text {hvp }}$ or the vacuum polarization one is using. We will compare our predictions quantitatively to the leading prediction of chiral perturbation theory.

The only input required in our analysis is the timelike pion form factor, including its phase, which coincides with the iso-vector $p$-wave $\pi \pi$ scattering phase. We use the phenomenologically successful Gounaris-Sakurai (GS, [92]) parameterization of the form factor as described in [54], noting that alternative parameterizations are available (see [97] and references therein). Clearly, the most important feature in the form factor is the $\rho$ resonance. The main finite-size effect is that the finite-volume correlator falls off more rapidly than its infinite-volume counterpart, because the finite-volume spectrum is discrete and starts at a higher energy than $2 m_{\pi}$.

In order to proceed, we separate the correlator into two parts, $t<t_{i}$ and $t>t_{i}$, with $t_{i} \approx 1 \mathrm{fm}$. The reason for doing so is that the long-distance part can be analyzed using the low-lying energy-eigenstates on the torus. At shorter distances, the Poisson-resummed expression based on non-interacting pions should provide a good approximation to the finite-size effects for realistic $m_{\pi} L \geq 4$ [54]. As we show below, the finite-volume effects for the contribution to $a_{\mu}$ from $t<1 \mathrm{fm}$ are negligible for $m_{\pi} L \geq 4$ and $m_{\pi} \lesssim 300 \mathrm{MeV}$. 


\begin{tabular}{|ccccccc|}
\hline Run & $M_{\pi}[\mathrm{MeV}]$ & $m_{\rho}[\mathrm{MeV}]$ & $\Gamma_{\rho}[\mathrm{MeV}]$ & $M_{\pi} L$ & $t_{i}[\mathrm{fm}]$ & $m_{\text {eff }}(1 \mathrm{fm}, L)[\mathrm{MeV}]$ \\
\hline P4 & 139.57 & 773 & 130 & 4.0 & 1.41 & 734 \\
\hline A5 & 331 & 912 & 61 & 4.0 & 0.60 & 927 \\
B6 & 281 & 852 & 75 & 5.0 & 1.10 & 854 \\
\hline F6 & 311 & 879 & 64 & 5.0 & 0.99 & 885 \\
F7 & 265 & 834 & 80 & 4.2 & 0.82 & 837 \\
G8 & 185 & 790 & 113 & 4.0 & 1.07 & 770 \\
\hline N6 & 341 & 910 & 55 & 4.0 & 0.58 & 928 \\
O7 & 268 & 835 & 79 & 4.4 & 0.89 & 838 \\
\hline
\end{tabular}

Table 9. Parameters of the Gounaris-Sakurai model used to explore finite-size effects on the various ensembles. $\mathrm{P} 4$ is a hypothetical ensemble at the physical pion mass. The width parameter at the physical pion mass is taken from [54], and is estimated from there for the other pion masses according to $\Gamma_{\rho} \propto k_{\rho}^{3} / m_{\rho}^{2}, k_{\rho} \equiv \frac{1}{2}\left(m_{\rho}^{2}-4 M_{\pi}^{2}\right)^{1 / 2}$. We chose $t_{i}=\left(m_{\pi} L / 4\right)^{2} / m_{\pi}$.

Specifically, we define the short- and long-distance contributions

$$
a_{\mu}^{\mathrm{hvp}}(L)=a_{\mu}^{<}\left(t_{i}, L\right)+a_{\mu}^{>}\left(t_{i}, L\right)
$$

computed on a finite torus as follows,

$$
a_{\mu}^{<}\left(t_{i}, L\right) \equiv\left(\frac{\alpha}{\pi}\right)^{2} \int_{0}^{t_{i}} d t G(t, L) \tilde{f}(t), \quad a_{\mu}^{>}\left(t_{i}, L\right) \equiv\left(\frac{\alpha}{\pi}\right)^{2} \int_{t_{i}}^{\infty} d t G(t, L) \tilde{f}(t) .
$$

Here $\tilde{f}(t)$ is the QED kernel, given explicitly in appendix B. The Euclidean time $t_{i}$ represents the point beyond which the two-pion channel dominates the correlator.

Using the Gounaris-Sakurai model combined with the Lüscher formalism for $a_{\mu}^{>}$, as in [54], we obtain for the sets of parameters listed in table 9 the estimates of the finite-size effects in table 10. The effects are sizeable compared to the ultimate sub-percent accuracy goal. In addition to the lattice ensembles available to us, we also consider for illustration an ensemble at the physical pion mass and $m_{\pi} L=4$, labelled P4. For $a_{\mu}^{<}$, we use the free-pion approximation to compute finite-size effects. Some details of this approximation are given in the next subsection.

\section{C.1 Finite-volume corrections for non-interacting pions}

For non-interacting pions, finite-size effects can be obtained by an elementary computation. We use eqs. (A.13)-(A.14) of [54], which can be written in terms of a non-oscillating integrand as follows,

$$
\begin{aligned}
G(t, L)-G(t, \infty) \stackrel{t \geq 0}{=} & \frac{1}{3}\left[\frac{1}{L^{3}} \sum_{\vec{k}}-\int \frac{d^{3} k}{(2 \pi)^{3}}\right] \frac{\vec{k}^{2}}{\vec{k}^{2}+m_{\pi}^{2}} e^{-2 t \sqrt{\vec{k}^{2}+m_{\pi}^{2}}} \\
= & \frac{m_{\pi}^{4} t}{3 \pi^{2}} \sum_{\vec{n} \neq 0}\left\{\frac{K_{2}\left(m_{\pi} \sqrt{L^{2} \vec{n}^{2}+4 t^{2}}\right)}{m_{\pi}^{2}\left(L^{2} \vec{n}^{2}+4 t^{2}\right)}\right. \\
& \left.-\frac{1}{m_{\pi} L|\vec{n}|} \int_{1}^{\infty} d y K_{0}\left(m_{\pi} y \sqrt{L^{2} \vec{n}^{2}+4 t^{2}}\right) \sinh \left(m_{\pi} L|\vec{n}|(y-1)\right)\right\} .
\end{aligned}
$$




\begin{tabular}{|c|c|c|c|c|c|}
\hline Run & $a_{\mu}(\infty)$ & $a_{\mu}^{>}\left(t_{i}, \infty\right)$ & $\begin{array}{r}a_{\mu}^{<}\left(t_{i}, \infty\right) \\
-a_{\mu}^{<}\left(t_{i}, L\right)\end{array}$ & $\begin{array}{c}a_{\mu}^{>}\left(t_{i}, \infty\right) \\
-a_{\mu}^{>}\left(t_{i}, L\right)\end{array}$ & $\begin{array}{c}a_{\mu}^{>}\left(t_{i}, \infty\right) \\
-a_{\mu}^{>}{ }^{\mathrm{xpol}}\left(t_{i}, t_{f}, t_{\mathrm{cut}}, L\right)\end{array}$ \\
\hline P4 & 478 & 201 & 1.7 & 18.7 & 48.3 \\
\hline A5 & 260 & 218 & 0.32 & 11.1 & 11.6 \\
B6 & 305 & 142 & 0.61 & 4.3 & 6.9 \\
\hline F6 & 280 & 146 & 0.50 & 4.1 & 5.6 \\
F7 & 321 & 229 & 0.55 & 10.3 & 12.7 \\
G8 & 408 & 241 & 0.98 & 15.0 & 26.0 \\
\hline N6 & 253 & 216 & 0.30 & 11.3 & 11.7 \\
O7 & 316 & 207 & 0.58 & 8.4 & 10.9 \\
\hline
\end{tabular}

Table 10. Estimates of the finite-size effects on $a_{\mu}^{\text {hvp }}$ in the TMR in units of $10^{-10}$, based on non-interacting pions for the 'short-distance' contribution $a_{\mu}^{<}$and on the Gounaris-Sakurai model of the timelike pion form factor and the Lüscher formalism for the 'long-distance' contribution $a_{\mu}$. The last column is discussed in section C.3. We used the values $t_{i}=\left(m_{\pi} L / 4\right)^{2} / m_{\pi}, t_{f}=1 \mathrm{fm}$ and $t_{\text {cut }}=\max \left(t_{i}, 1.35 \mathrm{fm}\right)$. The parameters used for the different ensembles are listed in table 9.

We compute the finite-size effect from the part $t<t_{i}$ using eq. (C.4) and obtain the values quoted in table 10, column 4 . The small values indicate that the finite-size effects from the region below about $1 \mathrm{fm}$ can be neglected for $m_{\pi} \lesssim 300 \mathrm{MeV}$ and for $m_{\pi} L \geq 4$.

If we compute the finite-size effect at large Euclidean times using free pions (using eq. (C.3)), we obtain for instance

$$
10^{10} \cdot\left[a_{\mu}^{>}\left(t_{i}, \infty\right)-a_{\mu}^{>}\left(t_{i}, L\right)\right]=\left\{\begin{array}{cc}
12.6 & \left(\mathrm{P} 4, t_{i}=1.41 \mathrm{fm}\right) \\
8.0 & \left(\mathrm{G} 8, t_{i}=1.07 \mathrm{fm}\right)
\end{array}\right.
$$

We see that, although of the same order of magnitude as the finite-size effects in table 10 (column 5) estimated using the Gounaris-Sakurai model in conjunction with the Lüscher formalism, the numbers in eq. (C.5) are smaller by a factor 1.5-2.0. For any fixed $t$, we expect the free-pion theory to predict the leading finite-size effect $\left(\mathrm{O}\left(e^{-m_{\pi} L}\right)\right)$ for $L$ sufficiently large. However, at times $t>1 \mathrm{fm}$, many terms contribute significantly in the winding expansion eq. (C.4) at realistic parameters. It is then more expedient to use the sum over energy eigenstates as in eq. (C.3), however, with the energy levels and matrix elements taking into account $\pi \pi$ interactions via the Lüscher formalism. We conclude that the interactions between pions play an important role in estimating the finite-size effect in the $t>1 \mathrm{fm}$ region at the typical volumes $m_{\pi} L \approx 4$.

The Gounaris-Sakurai model also allows us to estimate a lower bound on the value of $m_{\pi} L$ for which finite-size effects in $a_{\mu}^{\text {hvp }}$ are below the level of $1 \%$. From table 10 we can read off that finite-size effects from the region $t>1.4 \mathrm{fm}$ are as large as $3 \%$ for ensemble $\mathrm{P} 4$. By repeating the analysis for larger values of $m_{\pi} L$ we find that finite-size effects from the region $t>1.4 \mathrm{fm}$ are reduced to about $1 \%$ when $m_{\pi} L \approx 6$. By contrast, finite-size effects from the region below $1.4 \mathrm{fm}$ are already well below $1 \%$ for $m_{\pi} L=4$. 


\begin{tabular}{|c|c|}
\hline G8: parameter varied & $10^{10}\left(a_{\mu}(\infty)-a_{\mu}(L)\right)$ \\
\hline$t_{i}=1.2 \mathrm{fm}$ & 15.8 \\
$t_{i}=0.9 \mathrm{fm}$ & 16.1 \\
\hline$m_{\rho}=780 \mathrm{MeV}$ & 16.2 \\
$m_{\rho}=800 \mathrm{MeV}$ & 15.8 \\
\hline$\Gamma_{\rho}=90 \mathrm{MeV}$ & 16.0 \\
$\Gamma_{\rho}=136 \mathrm{MeV}$ & 16.0 \\
\hline
\end{tabular}

Table 11. Change in the size of the finite-volume effect under variations of the parameters. Only one parameter is varied at a time. The default values of the parameters are those given in table 9; they lead to $a_{\mu}(\infty)-a_{\mu}(L)=16.0 \cdot 10^{-10}$ (sum of column 4 and 5 in table 10$)$.

\section{C.2 Reliability of the estimate of finite-size effects}

To discuss the dependence of our theory estimate of the finite-size effect on the parameters, we focus on the ensemble G8, where the correction is sizeable. Using the GS model combined with the Lüscher formalism, we obtain

$$
t \cdot\left(\frac{\alpha}{\pi}\right)^{2}(G(t, \infty)-G(t, L)) \tilde{f}(t)=4.4 \cdot 10^{-10}
$$

at $t=t_{i}=1.07 \mathrm{fm}$, while for free pions, we get for the same quantity $3.3 \cdot 10^{-10}$. Thus at the turning point, where we switch from the free-pion to the interacting-pion case, the difference between the two predictions is moderate. This is a first indication that the overall prediction of the finite-size effect is not too sensitive to the turning point $t_{i}$. Explicitly, we explore the dependence of the predicted finite-size effect on various parameters in table 11. The result hardly changes under reasonable variations of $t_{i}, m_{\rho}$ and $\Gamma_{\rho}$. Of course the small observed variations do not reflect the full uncertainty due to the use of the GounarisSakurai parameterization, the corrections to the finite-size effect at $t<t_{i}$ due to pion interactions and internal structure, etc. We think that the genuine finite-size effects on $a_{\mu}^{\text {hvp }}$ (i.e. the sum of column 4 and 5 in table 10) are correctly estimated to within $20 \%$ in our approach.

We have also performed a sanity check by comparing our prediction for finite-size effects to the direct lattice QCD data in [39], where at one set of quark masses, results for $a_{\mu}^{\text {hvp }}$ at three volumes are available: within the uncertainties, our estimate for the volumedependence of $\left.\frac{d \Pi}{d Q^{2}}\right|_{Q^{2}=0}$ is fully consistent with the numerical data. In the comparison, we assume that finite-size effects are dominated by the iso-vector contribution to $a_{\mu}^{\text {hvp }}$, since the iso-scalar $\omega$ and $\phi$ resonances are extremely narrow.

\section{C.3 Single-exponential extension of the time-momentum correlator}

Since in practice an extension of the vector correlator is used at long distances, we introduce

$$
a_{\mu}^{>\text {xpol }}\left(t_{i}, t_{f}, t_{\mathrm{cut}}, L\right) \equiv\left(\frac{\alpha}{\pi}\right)^{2}\left\{\int_{t_{i}}^{t_{\mathrm{cut}}} d t G(t, L) \tilde{f}(t)+\int_{t_{\mathrm{cut}}}^{\infty} d t G_{\mathrm{xpol}}\left(t ; t_{f}, L\right) \tilde{f}(t)\right\}
$$


where $t_{\text {cut }}>t_{i}$ is the point beyond which the one-exponential extrapolation of the finitevolume correlator

$$
G_{\mathrm{xpol}}\left(t ; t_{f}, L\right) \equiv A_{\mathrm{eff}}\left(t_{f}, L\right) e^{-m_{\mathrm{eff}}\left(t_{f}, L\right) t}
$$

is used, based on the effective mass and amplitude determined at time $t_{f}$; explicitly,

$$
m_{\mathrm{eff}}(t, L) \equiv-\frac{d}{d t} \log G(t, L), \quad A_{\mathrm{eff}}(t, L) \equiv G(t, L) e^{m_{\mathrm{eff}}(t, L)} .
$$

The reason for considering $a_{\mu}^{>\text {xpol }}\left(t_{i}, t_{f}, t_{\text {cut }}, L\right)$ is that due to the deteriorating signal-tonoise ratio on the vector correlator at large distances, some form of extrapolation is required in practice to be able to integrate to $t=\infty$.

We indicate in the last column of table 10 what error one incurs by replacing the correlator by its one-exponential extension beyond $t_{\text {cut }}$. As compared to the genuine finitesize effect (column 5 of the table), the additional systematic error is relatively modest until one reaches the ensembles with $m_{\pi}$ below $200 \mathrm{MeV}$. At this point, the result is also quite sensitive to the time $t_{f}$ where the effective mass is determined. On ensemble G8 for instance, we obtain

$$
\begin{aligned}
& 10^{10} \cdot\left(a_{\mu}(\infty)-\left[a_{\mu}^{<}\left(t_{i}, L\right)+a^{>, \mathrm{xpol}}\left(t_{i}, t_{f}, t_{\mathrm{cut}}, L\right)\right]\right) \\
& = \begin{cases}31.1 & t_{f}=0.85 \mathrm{fm}, m_{\mathrm{eff}}\left(t_{f}, L\right)=777 \mathrm{MeV}, \\
23.9 & t_{f}=1.15 \mathrm{fm}, m_{\mathrm{eff}}\left(t_{f}, L\right)=764 \mathrm{MeV} .\end{cases}
\end{aligned}
$$

Thus for ensembles with $m_{\pi} \lesssim 200 \mathrm{MeV}$, the single-exponential extension is clearly inadequate once the precision goal on $a_{\mu}^{\text {hvp }}$ is $5 \%$ or better.

\section{C.4 Uncertainty in the determination of the $\rho$-mass and decay width}

In the absence of a full dedicated study of the spectroscopy in the iso-vector vector channel, in section 4.2 we have assumed the GS form of the timelike pion form factor and used a simplified procedure to determine the parameters $\left(m_{\rho}, \Gamma_{\rho}\right)$ of the model. On our ensemble G8 with the lightest pion mass, we assumed that the ground state had an energy of $E_{0}=$ $2 \sqrt{m_{\pi}^{2}+(2 \pi / L)^{2}}$ corresponding to non-interacting pions in a $p$-wave, while the energy of the first excited state was identified with the parameter $m_{\rho}$ of the GS model. We have investigated how reliable these assumptions are using the GS model; see figure 5. Especially the first excited state corresponds to the $\rho$-mass to sub-percent accuracy for a wide range of parameters. The deviation of the ground state from the non-interacting-pions predictions is at the $3-4 \%$ level. At our present level of accuracy, this is a sufficient level of control to avoid a significant bias in the determination of the first excited state, since the ground state contributes with a relatively weak amplitude to the vector correlator.

\section{Determination of the quark-disconnected contribution}

In this appendix we provide the details of our calculation of the quark-disconnected contribution to $a_{\mu}^{\text {hvp }}$, which has been performed using the TMR formulation (see also contribution 2.16 in [98]). Analytic analyses of disconnected contributions have been presented 

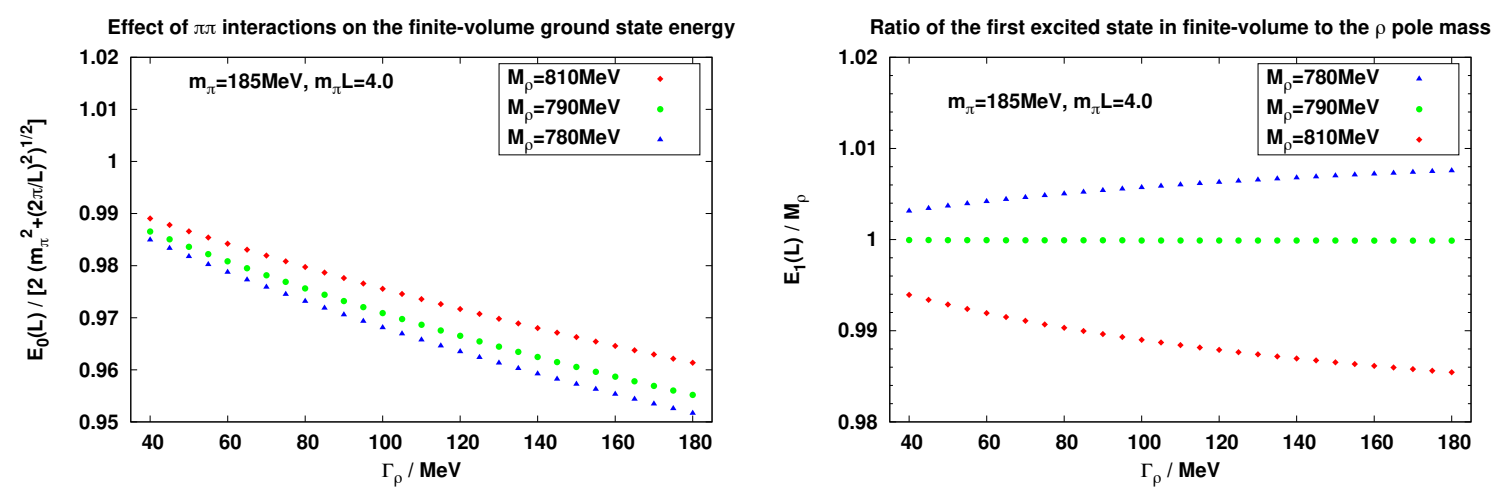

Figure 5. Corrections to energy levels relative to the naive expectation of a non-interacting, $p$-wave two-pion state and a $\rho$-state, for parameters corresponding to ensemble G8 and assuming the GS pion form factor. Left: correction to the expectation $E_{0}=2 \sqrt{m_{\pi}^{2}+(2 \pi / L)^{2}}$ for the ground-state energy as a function of the width $\Gamma_{\rho}$, for three values of the mass $m_{\rho}$. Right: correction to the expectation $E_{1}=m_{\rho}$.

in $[99,100]$. For our discussion it is useful to recall the expression for $a_{\mu}^{\text {hvp }}$ in the TMR, i.e.

$$
a_{\mu}^{\mathrm{hvp}}=\left(\frac{\alpha}{\pi}\right)^{2} \int_{0}^{\infty} d x_{0} G\left(x_{0}\right) \widetilde{K}\left(x_{0} ; m_{\mu}\right)
$$

where $\widetilde{K}\left(x_{0} ; m_{\mu}\right)$ is defined in eq. (2.8). In the following we restrict the analysis to the contributions from the $u, d$ and $s$ quarks only, so that the electromagnetic current is given by

$$
J_{\mu}(x)=\frac{2}{3} \bar{u}(x) \gamma_{\mu} u(x)-\frac{1}{3} \bar{d}(x) \gamma_{\mu} d(x)-\frac{1}{3} \bar{s}(x) \gamma_{\mu} s(x)
$$

After performing the Wick contractions one can identify the connected and disconnected parts as

$$
G\left(x_{0}\right)=G^{u d}\left(x_{0}\right)+G^{s}\left(x_{0}\right)-G_{\text {disc }}\left(x_{0}\right),
$$

where $G^{u d}$ and $G^{s}$ are defined according to eq. (3.7), and the total disconnected contribution $G_{\text {disc }}\left(x_{0}\right)$ is given by

$$
G_{\text {disc }}\left(x_{0}\right)=G_{\text {disc }}^{u d}\left(x_{0}\right)+G_{\text {disc }}^{s}\left(x_{0}\right)-2 G_{\text {disc }}^{u d, s}\left(x_{0}\right)
$$

The superscripts indicate whether the contribution involves only light $(u d)$, strange $(s)$ or both $(u d, s)$ quark flavours (note that we work in the isospin limit, $m_{u}=m_{d}$ ).

In ref. [35] it was shown that $G_{\text {disc }}\left(x_{0}\right)$ factorizes according to

$$
G_{\text {disc }}\left(x_{0}\right)=-\frac{1}{9}\left\langle\left(\Delta^{u d}\left(x_{0}\right)-\Delta^{s}\left(x_{0}\right)\right)\left(\Delta^{u d}(0)-\Delta^{s}(0)\right)\right\rangle
$$

where $\Delta^{f}\left(x_{0}\right)$ for $f=(u d), s$ is given by

$$
\Delta^{f}\left(x_{0}\right)=\int d^{3} x \operatorname{Tr}\left[\gamma_{k} S^{f}(x, x)\right],
$$




\begin{tabular}{|cccccc|}
\hline Run & $N_{\text {cfg }}$ & $N_{\mathrm{r}}$ & $T / a$ & $x_{0}^{*}$ & $\Delta a_{\mu}^{\text {hvp }}$ \\
\hline E5 & 1000 & 75 & 64 & 25 & $0.7 \%$ \\
& & & & 28 & $0.3 \%$ \\
\hline F6 & \multirow{2}{*}{300} & \multirow{2}{*}{45} & 96 & 22 & $1.8 \%$ \\
& & & & 23 & $1.5 \%$ \\
\hline
\end{tabular}

Table 12. Details of the evaluation of quark-disconnected contribution $G_{\text {disc }}\left(x_{0}\right)$ (see eq. (D.3)). $N_{\mathrm{r}}$ denotes the number of stochastic sources per timeslice, while $x_{0}^{*}$ represents the Euclidean time at which the ratio $G_{\mathrm{disc}}\left(x_{0}\right) / C^{\rho \rho}\left(x_{0}\right)$ is replaced by its asymptotic value. The upper bound on the size of the quark-disconnected contribution to $a_{\mu}^{\text {hvp }}$ is given by $\Delta a_{\mu}^{\text {hvp }}$.

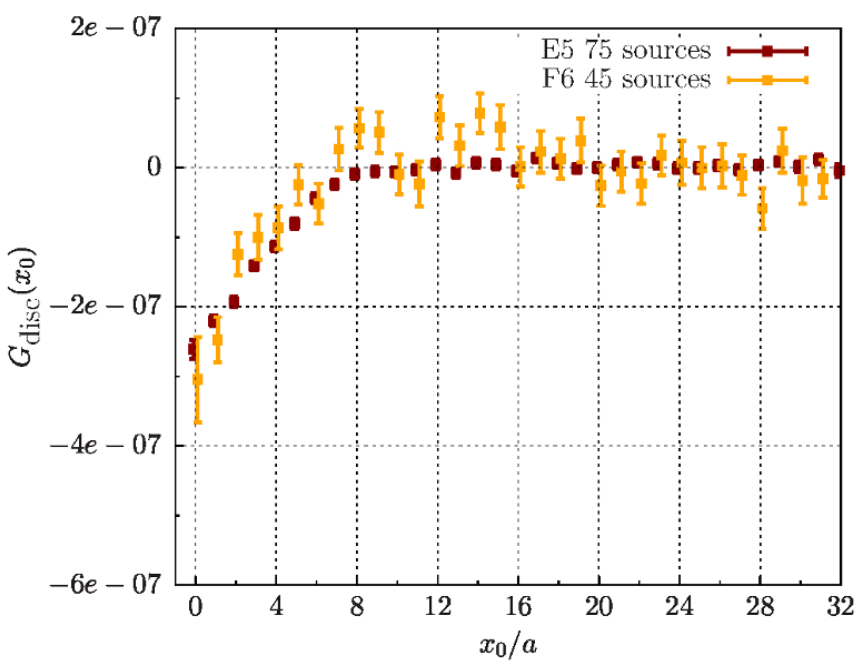

Figure 6. The quark-disconnected contribution $G_{\text {disc }}\left(x_{0}\right)$ to the vector correlator (in lattice units) computed on ensembles E5 and F6.

and $S^{f}$ denotes the quark propagator of flavour $f$. Statistically accurate results for quantities such as $\Delta^{f}$ require "all-to-all" propagators which are commonly computed using stochastic noise sources. In [35] it was shown that the statistical accuracy of $G_{\text {disc }}\left(x_{0}\right)$ can be significantly enhanced when $\Delta^{u d}$ and $\Delta^{s}$ are computed using the same random noise vectors, since the correlations between the light and strange quark contributions largely cancel the stochastic noise.

In our determination of $G_{\text {disc }}\left(x_{0}\right)$ we have used stochastic sources in conjunction with a hopping parameter expansion (HPE) of the quark propagator [101], suitably adapted to the case of $\mathrm{O}(a)$ improved Wilson quarks [102]. The calculation was performed at our intermediate value of the lattice spacing at pion masses of 437 and $311 \mathrm{MeV}$, respectively (ensembles E5 and F6). The all-to-all propagators for the light and strange quarks were computed by employing a 6th order HPE in combination with $N_{\mathrm{r}}$ stochastic U(1) noise vectors $\eta_{k}(\vec{x}), k=1, \ldots, N_{\mathrm{r}}$ on each timeslice. Further details are listed in table 12.

Results for $G_{\text {disc }}\left(x_{0}\right)$ on the two ensembles under study are shown in figure 6 . While a small but non-zero signal is observed for $x_{0} / a \lesssim 8$ the disconnected contribution $G_{\text {disc }}\left(x_{0}\right)$ 
vanishes within errors for larger values of $x_{0}$. At small times the disconnected contribution is only about $0.005 \%$ of the connected one, and hence we conclude that the vector correlator $G\left(x_{0}\right)$ is completely dominated by the connected part in the region $x_{0} \lesssim 0.5 \mathrm{fm}$.

The fact that the disconnected contribution is small where it can be resolved does not, however, imply that it is negligible. Using our data we can derive an upper bound on the error which arises if one were to neglect the disconnected contribution altogether. To this end it is useful to recall the isospin decomposition of the electromagnetic current shown in eq. (2.13), which gives rise to the iso-vector $(I=1)$ correlator $G^{\rho \rho}$ and its iso-scalar counterpart $G^{I=0}$ (see eq. $(2.15)$ ). The iso-vector correlator $G^{\rho \rho}\left(x_{0}\right)$ contains only quark-connected diagrams; it is related to the connected light quark contribution $G^{u d}\left(x_{0}\right)$ via

$$
G^{\rho \rho}\left(x_{0}\right)=\frac{9}{10} G^{u d}\left(x_{0}\right) .
$$

By contrast, the iso-scalar correlator $G^{I=0}$ contains both connected and disconnected contributions, i.e.

$$
G\left(x_{0}\right)^{I=0}=\frac{1}{10} G^{u d}\left(x_{0}\right)+G^{s}\left(x_{0}\right)-G_{\text {disc }}\left(x_{0}\right) .
$$

With the help of eqs. (D.3) and (D.7) one derives the expression

$$
-\frac{G_{\text {disc }}\left(x_{0}\right)}{G^{\rho \rho}\left(x_{0}\right)}=\frac{G\left(x_{0}\right)-G^{\rho \rho}\left(x_{0}\right)}{G^{\rho \rho}\left(x_{0}\right)}-\frac{1}{9}\left(1+9 \frac{G^{s}\left(x_{0}\right)}{G^{\rho \rho}\left(x_{0}\right)}\right) .
$$

It is now important to realize that the iso-scalar spectral function vanishes below the three-pion threshold, which implies that $G^{I=0}\left(x_{0}\right)=\mathrm{O}\left(\mathrm{e}^{-3 m_{\pi} x_{0}}\right)$ for $x_{0} \rightarrow \infty$. According to eq. (D.8) this implies

$$
\begin{aligned}
& G_{\mathrm{disc}}\left(x_{0}\right)=\left(\frac{1}{10} G^{u d}\left(x_{0}\right)+G^{s}\left(x_{0}\right)\right) \cdot\left(1+\mathrm{O}\left(e^{-m_{\pi} x_{0}}\right)\right), \\
& G\left(x_{0}\right)=G^{\rho \rho}\left(x_{0}\right) \cdot\left(1+\mathrm{O}\left(e^{-m_{\pi} x_{0}}\right)\right)
\end{aligned}
$$

in the deep infrared. With these considerations one determines the asymptotic behaviour of the ratio in eq. (D.9) in the long-distance regime as

$$
-\frac{G_{\text {disc }}\left(x_{0}\right)}{G^{\rho \rho}\left(x_{0}\right)} \stackrel{x_{0} \rightarrow \infty}{\longrightarrow}-\frac{1}{9}
$$

where we have also taken into account that $G^{s}\left(x_{0}\right)$ drops off faster than $G^{\rho \rho}\left(x_{0}\right)$ due to the heavier mass of the strange quark. We expect the asymptotic value to be approached from above, because $\left[G\left(x_{0}\right)-G^{\rho \rho}\left(x_{0}\right)\right] \sim \frac{1}{18} \mathrm{e}^{-m_{\omega} x_{0}}$ is likely larger than $G^{s}\left(x_{0}\right) \sim \frac{1}{9} \mathrm{e}^{-m_{\phi} x_{0}}$ for $x_{0} \gtrsim 1 \mathrm{fm}$.

In figure 7 we plot the ratio of eq. (D.9) versus the Euclidean distance. One can see that the ratio is practically zero up to $x_{0} / a \approx 26$ on E5 and $x_{0} / a \approx 22$ at the smaller pion mass of ensemble F6. Thus, there is no visible trend for distances $x_{0} \lesssim 1.7 \mathrm{fm}$ that the ratio approaches its asymptotic value of $-1 / 9$. In order to derive a conservative upper bound on the quark-disconnected contribution we assume that the ratio of eq. (D.9) drops 

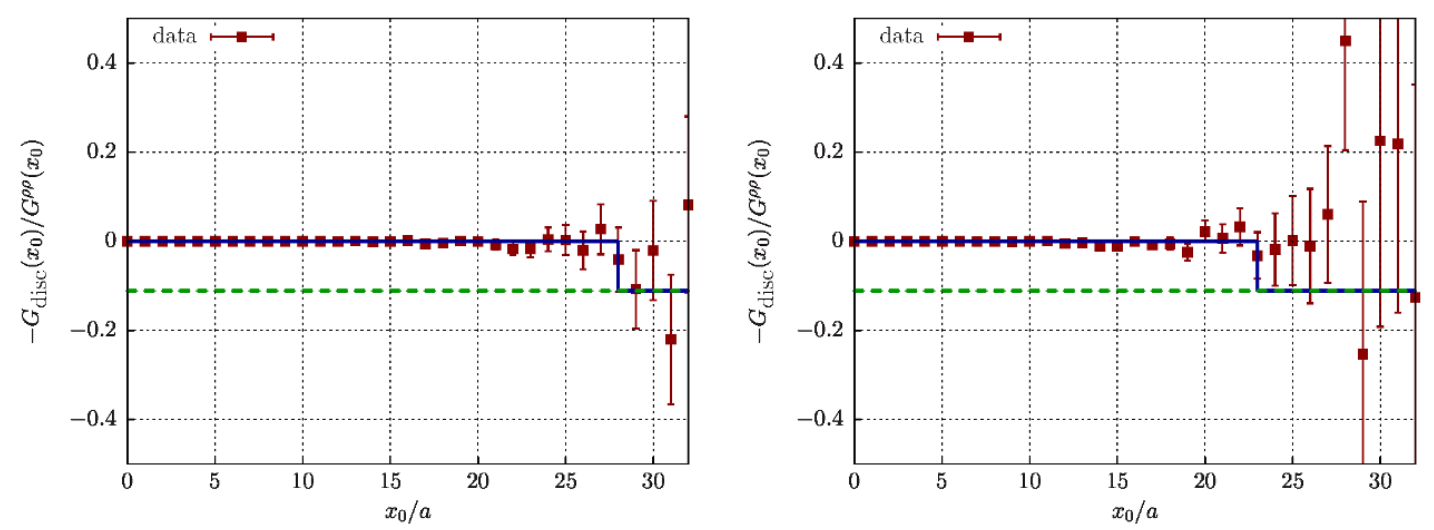

Figure 7. The ratio of the disconnected to the (connected) iso-vector contribution to the vector correlator for ensembles E5 (left) and F6 (right).

to $-1 / 9$ at the time $x_{0}^{*}$ where the accuracy of the data is insufficient to distinguish between zero and the expected asymptotic value. In other words, we set

$$
-\frac{G_{\text {disc }}\left(x_{0}\right)}{G^{\rho \rho}\left(x_{0}\right)}=\left\{\begin{array}{cc}
0, & x_{0} \leq x_{0}^{*}, \\
-1 / 9, & x_{0}>x_{0}^{*}
\end{array}\right.
$$

If we write the hadronic vacuum polarization contribution $a_{\mu}^{\text {hvp }}$ as the sum of the quarkconnected and -disconnected contributions, $a_{\mu}^{\text {hvp }}=\left(a_{\mu}^{\text {hvp }}\right)_{\text {con }}+\left(a_{\mu}^{\text {hvp }}\right)_{\text {disc }}$, we can define

$$
\Delta a_{\mu}^{\text {hvp }}:=\frac{\left(a_{\mu}^{\text {hvp }}\right)_{\text {con }}-a_{\mu}^{\text {hvp }}}{\left(a_{\mu}^{\text {hvp }}\right)_{\text {con }}} \equiv-\frac{\left(a_{\mu}^{\text {hvp }}\right)_{\text {disc }}}{\left(a_{\mu}^{\text {hvp }}\right)_{\text {con }}},
$$

which is the relative size of the disconnected and connected contributions, and $\left(a_{\mu}^{\text {hvp }}\right)_{\text {disc }}$ is given by

$$
\left(a_{\mu}^{\mathrm{hvp}}\right)_{\mathrm{disc}}=\left(\frac{\alpha}{\pi}\right)^{2} \int_{0}^{\infty} d x_{0}\left(-G_{\mathrm{disc}}\left(x_{0}\right)\right) \widetilde{K}\left(x_{0} ; m_{\mu}\right) .
$$

After inserting eqs. (D.13) and (D.7) we obtain the maximum estimate of the quarkdisconnected contribution as

$$
\left(a_{\mu}^{\mathrm{hvp}}\right)_{\mathrm{disc}}=-\frac{1}{10}\left(\frac{\alpha}{\pi}\right)^{2} \int_{x_{0}^{*}}^{\infty} d x_{0} G^{u d}\left(x_{0}\right) \widetilde{K}\left(x_{0} ; m_{\mu}\right) .
$$

The resulting estimates for the relative contribution $\Delta a_{\mu}^{\text {hvp }}$ are listed in table 12 .

Open Access. This article is distributed under the terms of the Creative Commons Attribution License (CC-BY 4.0), which permits any use, distribution and reproduction in any medium, provided the original author(s) and source are credited.

\section{References}

[1] Particle Data Group collaboration, C. Patrignani et al., Review of Particle Physics, Chin. Phys. C 40 (2016) 100001 [InSPIRE].

[2] S. Eidelman and F. Jegerlehner, Hadronic contributions to $g-2 o f$ the leptons and to the effective fine structure constant $\alpha\left(M_{Z}^{2}\right), Z$. Phys. C 67 (1995) 585 [hep-ph/9502298] [INSPIRE]. 
[3] M. Davier, A. Hoecker, B. Malaescu and Z. Zhang, Reevaluation of the Hadronic Contributions to the Muon $g-2$ and to $\alpha\left(M_{Z}\right)$, Eur. Phys. J. C 71 (2011) 1515 [Erratum ibid. C 72 (2012) 1874] [arXiv: 1010.4180] [INSPIRE].

[4] K. Hagiwara, R. Liao, A.D. Martin, D. Nomura and T. Teubner, $(g-2)_{\mu}$ and $\alpha\left(M_{Z}^{2}\right)$ re-evaluated using new precise data, J. Phys. G 38 (2011) 085003 [arXiv:1105.3149] [INSPIRE].

[5] T. Blum et al., The Muon $(g-2)$ Theory Value: Present and Future, arXiv:1311.2198 [INSPIRE].

[6] A.V. Nesterenko, Dispersive approach to $Q C D$ and hadronic contributions to electroweak observables, EPJ Web Conf. 137 (2017) 05021 [arXiv: 1701.00678] [INSPIRE].

[7] F. Jegerlehner, Muon g-2 Theory: the Hadronic Part, arXiv:1705.00263 [INSPIRE].

[8] C.M. Carloni Calame, M. Passera, L. Trentadue and G. Venanzoni, A new approach to evaluate the leading hadronic corrections to the muon g-2, Phys. Lett. B 746 (2015) 325 [arXiv: 1504. 02228] [INSPIRE].

[9] G. Abbiendi et al., Measuring the leading hadronic contribution to the muon $g-2$ via $\mu$ e scattering, Eur. Phys. J. C 77 (2017) 139 [arXiv:1609.08987] [InSPIRE].

[10] E. de Rafael, Moment Analysis of Hadronic Vacuum Polarization - Proposal for a lattice QCD evaluation of $g_{\mu}-2$, Phys. Lett. B 736 (2014) 522 [arXiv:1406.4671] [InSPIRE].

[11] E. de Rafael, Hadronic vacuum polarization in $Q C D$ and its evaluation in Euclidean spacetime, Phys. Rev. D 96 (2017) 014510 [arXiv:1702.06783] [InSPIRE].

[12] M. Benayoun, P. David, L. DelBuono and F. Jegerlehner, A BHLS model based moment analysis of muon $g-2$ and its use for lattice $Q C D$ evaluations of $a_{\mu}^{\text {had }}$, arXiv: 1605.04474 [INSPIRE].

[13] S. Bodenstein, C.A. Dominguez and K. Schilcher, Hadronic contribution to the muon $g-2$ factor: A theoretical determination, Phys. Rev. D 85 (2012) 014029 [arXiv:1106.0427] [INSPIRE].

[14] C.A. Dominguez, K. Schilcher and H. Spiesberger, QCD determination of the leading order hadronic contribution to the muon $g-2$, arXiv:1704.02843 [INSPIRE].

[15] F. Jegerlehner and A. Nyffeler, The Muon g-2, Phys. Rept. 477 (2009) 1 [arXiv:0902.3360] [INSPIRE].

[16] J. Prades, E. de Rafael and A. Vainshtein, The Hadronic Light-by-Light Scattering Contribution to the Muon and Electron Anomalous Magnetic Moments, Adv. Ser. Direct. High Energy Phys. 20 (2009) 303 [arXiv:0901.0306] [INSPIRE].

[17] J. Bijnens, Hadronic light-by-light contribution to $a_{\mu}$ : extended Nambu-Jona-Lasinio, chiral quark models and chiral Lagrangians, EPJ Web Conf. 118 (2016) 01002 [arXiv: 1510.05796] [INSPIRE].

[18] V. Pascalutsa and M. Vanderhaeghen, Sum rules for light-by-light scattering, Phys. Rev. Lett. 105 (2010) 201603 [arXiv: 1008.1088] [INSPIRE].

[19] V. Pascalutsa, V. Pauk and M. Vanderhaeghen, Light-by-light scattering sum rules constraining meson transition form factors, Phys. Rev. D 85 (2012) 116001 [arXiv: 1204.0740] [INSPIRE].

[20] V. Pauk and M. Vanderhaeghen, Two-loop massive scalar three-point function in a dispersive approach, arXiv:1403.7503 [INSPIRE]. 
[21] V. Pauk and M. Vanderhaeghen, Anomalous magnetic moment of the muon in a dispersive approach, Phys. Rev. D 90 (2014) 113012 [arXiv:1409.0819] [INSPIRE].

[22] I. Danilkin and M. Vanderhaeghen, Light-by-light scattering sum rules in light of new data, Phys. Rev. D 95 (2017) 014019 [arXiv:1611.04646] [InSPIRE].

[23] G. Colangelo, M. Hoferichter, M. Procura and P. Stoffer, Dispersive approach to hadronic light-by-light scattering, JHEP 09 (2014) 091 [arXiv: 1402.7081] [INSPIRE].

[24] G. Colangelo, M. Hoferichter, B. Kubis, M. Procura and P. Stoffer, Towards a data-driven analysis of hadronic light-by-light scattering, Phys. Lett. B 738 (2014) 6 [arXiv:1408.2517] [INSPIRE].

[25] G. Colangelo, M. Hoferichter, M. Procura and P. Stoffer, Dispersion relation for hadronic light-by-light scattering: theoretical foundations, JHEP 09 (2015) 074 [arXiv:1506.01386] [INSPIRE].

[26] G. Colangelo, M. Hoferichter, M. Procura and P. Stoffer, Rescattering effects in the hadronic-light-by-light contribution to the anomalous magnetic moment of the muon, Phys. Rev. Lett. 118 (2017) 232001 [arXiv:1701.06554] [INSPIRE].

[27] G. Colangelo, M. Hoferichter, M. Procura and P. Stoffer, Dispersion relation for hadronic light-by-light scattering: two-pion contributions, JHEP 04 (2017) 161 [arXiv:1702.07347] [INSPIRE].

[28] T. Blum, Lattice calculation of the lowest order hadronic contribution to the muon anomalous magnetic moment, Phys. Rev. Lett. 91 (2003) 052001 [hep-lat/0212018] [INSPIRE].

[29] C. Aubin and T. Blum, Calculating the hadronic vacuum polarization and leading hadronic contribution to the muon anomalous magnetic moment with improved staggered quarks, Phys. Rev. D 75 (2007) 114502 [hep-lat/0608011] [INSPIRE].

[30] X. Feng, K. Jansen, M. Petschlies and D.B. Renner, Two-flavor QCD correction to lepton magnetic moments at leading-order in the electromagnetic coupling, Phys. Rev. Lett. 107 (2011) 081802 [arXiv:1103.4818] [inSPIRE].

[31] P. Boyle, L. Del Debbio, E. Kerrane and J. Zanotti, Lattice Determination of the Hadronic Contribution to the Muon g-2 using Dynamical Domain Wall Fermions, Phys. Rev. D 85 (2012) 074504 [arXiv:1107.1497] [INSPIRE].

[32] M. Della Morte, B. Jäger, A. Jüttner and H. Wittig, Towards a precise lattice determination of the leading hadronic contribution to $(g-2)_{\mu}$, JHEP 03 (2012) 055 [arXiv:1112.2894] [INSPIRE].

[33] ETM collaboration, F. Burger, X. Feng, G. Hotzel, K. Jansen, M. Petschlies and D.B. Renner, Four-Flavour Leading-Order Hadronic Contribution To The Muon Anomalous Magnetic Moment, JHEP 02 (2014) 099 [arXiv: 1308.4327] [INSPIRE].

[34] HPQCD collaboration, B. Chakraborty et al., Strange and charm quark contributions to the anomalous magnetic moment of the muon, Phys. Rev. D 89 (2014) 114501 [arXiv:1403.1778] [INSPIRE].

[35] V. Gülpers, A. Francis, B. Jäger, H. Meyer, G. von Hippel and H. Wittig, The leading disconnected contribution to the anomalous magnetic moment of the muon, PoS (LATTICE2014) 128 [arXiv:1411.7592] [INSPIRE]. 
[36] T. Blum et al., Calculation of the hadronic vacuum polarization disconnected contribution to the muon anomalous magnetic moment, Phys. Rev. Lett. 116 (2016) 232002 [arXiv: 1512.09054] [INSPIRE].

[37] RBC/UKQCD collaboration, T. Blum et al., Lattice calculation of the leading strange quark-connected contribution to the muon g-2, JHEP 04 (2016) 063 [Erratum ibid. 05 (2017) 034] [arXiv:1602.01767] [INSPIRE].

[38] B. Chakraborty, C.T.H. Davies, J. Koponen, G.P. Lepage, M.J. Peardon and S.M. Ryan, Estimate of the hadronic vacuum polarization disconnected contribution to the anomalous magnetic moment of the muon from lattice QCD, Phys. Rev. D 93 (2016) 074509 [arXiv: 1512.03270] [INSPIRE].

[39] B. Chakraborty, C.T.H. Davies, P.G. de Oliviera, J. Koponen, G.P. Lepage and R.S. Van de Water, The hadronic vacuum polarization contribution to $a_{\mu}$ from full lattice QCD, Phys. Rev. D 96 (2017) 034516 [arXiv: 1601.03071] [INSPIRE].

[40] S. Borsányi et al., Slope and curvature of the hadron vacuum polarization at vanishing virtuality from lattice QCD, arXiv:1612.02364 [INSPIRE].

[41] S. Chowdhury, T. Blum, T. Izubuchi, M. Hayakawa, N. Yamada and T. Yamazaki, Calculating the light by light contribution to the muon anomalous magnetic moment using lattice QED, PoS(LATTICE 2008) 251.

[42] T. Blum, M. Hayakawa and T. Izubuchi, Update on the hadronic light-by-light contribution to the muon $g-2$ and inclusion of dynamically charged sea quarks, PoS (LATTICE 2013) 439.

[43] T. Blum, S. Chowdhury, M. Hayakawa and T. Izubuchi, Hadronic light-by-light scattering contribution to the muon anomalous magnetic moment from lattice QCD, Phys. Rev. Lett. 114 (2015) 012001 [arXiv: 1407.2923] [INSPIRE].

[44] T. Blum, N. Christ, M. Hayakawa, T. Izubuchi, L. Jin and C. Lehner, Lattice Calculation of Hadronic Light-by-Light Contribution to the Muon Anomalous Magnetic Moment, Phys. Rev. D 93 (2016) 014503 [arXiv:1510.07100] [INSPIRE].

[45] T. Blum et al., Connected and Leading Disconnected Hadronic Light-by-Light Contribution to the Muon Anomalous Magnetic Moment with a Physical Pion Mass, Phys. Rev. Lett. 118 (2017) 022005 [arXiv: 1610.04603] [INSPIRE].

[46] J. Green, O. Gryniuk, G. von Hippel, H.B. Meyer and V. Pascalutsa, Lattice QCD calculation of hadronic light-by-light scattering, Phys. Rev. Lett. 115 (2015) 222003 [arXiv: 1507.01577] [INSPIRE].

[47] J. Green et al., Direct calculation of hadronic light-by-light scattering, PoS (LATTICE 2015) 109 [arXiv: 1510.08384] [INSPIRE].

[48] N. Asmussen, J. Green, H.B. Meyer and A. Nyffeler, Position-space approach to hadronic light-by-light scattering in the muon $g-2$ on the lattice, PoS (LATTICE2016) 164 [arXiv: 1609.08454] [INSPIRE].

[49] A. Gérardin, H.B. Meyer and A. Nyffeler, Lattice calculation of the pion transition form factor $\pi^{0} \rightarrow \gamma^{*} \gamma^{*}$, Phys. Rev. D 94 (2016) 074507 [arXiv:1607.08174] [InSPIRE].

[50] E. de Rafael, Hadronic contributions to the muon g-2 and low-energy QCD, Phys. Lett. B 322 (1994) 239 [hep-ph/9311316] [INSPIRE].

[51] D. Bernecker and H.B. Meyer, Vector Correlators in Lattice QCD: Methods and applications, Eur. Phys. J. A 47 (2011) 148 [arXiv:1107.4388] [INSPIRE]. 
[52] C. Aubin, T. Blum, M. Golterman and S. Peris, Model-independent parametrization of the hadronic vacuum polarization and $g-2$ for the muon on the lattice, Phys. Rev. D 86 (2012) 054509 [arXiv:1205.3695] [INSPIRE].

[53] G.M. de Divitiis, R. Petronzio and N. Tantalo, On the extraction of zero momentum form factors on the lattice, Phys. Lett. B 718 (2012) 589 [arXiv:1208.5914] [INSPIRE].

[54] A. Francis, B. Jaeger, H.B. Meyer and H. Wittig, A new representation of the Adler function for lattice QCD, Phys. Rev. D 88 (2013) 054502 [arXiv:1306.2532] [INSPIRE].

[55] M. Golterman, K. Maltman and S. Peris, Tests of hadronic vacuum polarization fits for the muon anomalous magnetic moment, Phys. Rev. D 88 (2013) 114508 [arXiv:1309.2153] [INSPIRE].

[56] M. Golterman, K. Maltman and S. Peris, New strategy for the lattice evaluation of the leading order hadronic contribution to $(g-2)_{\mu}$, Phys. Rev. D 90 (2014) 074508 [arXiv:1405.2389] [INSPIRE].

[57] X. Feng, S. Hashimoto, G. Hotzel, K. Jansen, M. Petschlies and D.B. Renner, Computing the hadronic vacuum polarization function by analytic continuation, Phys. Rev. D 88 (2013) 034505 [arXiv: 1305.5878] [INSPIRE].

[58] S. Aoki et al., Review of lattice results concerning low-energy particle physics, Eur. Phys. J. C 77 (2017) 112 [arXiv: 1607.00299] [INSPIRE].

[59] B.e. Lautrup, A. Peterman and E. de Rafael, Recent developments in the comparison between theory and experiments in quantum electrodynamics, Phys. Rept. 3 (1972) 193 [INSPIRE].

[60] QCDSF collaboration, M. Göckeler, R. Horsley, W. Kürzinger, D. Pleiter, P.E.L. Rakow and G. Schierholz, Vacuum polarization and hadronic contribution to muon $g-2$ from lattice QCD, Nucl. Phys. B 688 (2004) 135 [hep-lat/0312032] [INSPIRE].

[61] G.M. de Divitiis, R. Petronzio and N. Tantalo, On the discretization of physical momenta in lattice QCD, Phys. Lett. B 595 (2004) 408 [hep-lat/0405002] [INSPIRE].

[62] C.T. Sachrajda and G. Villadoro, Twisted boundary conditions in lattice simulations, Phys. Lett. B 609 (2005) 73 [hep-lat/0411033] [INSPIRE].

[63] P.F. Bedaque and J.-W. Chen, Twisted valence quarks and hadron interactions on the lattice, Phys. Lett. B 616 (2005) 208 [hep-lat/0412023] [INSPIRE].

[64] C. Aubin, T. Blum, M. Golterman and S. Peris, Hadronic vacuum polarization with twisted boundary conditions, Phys. Rev. D 88 (2013) 074505 [arXiv:1307.4701] [INSPIRE].

[65] E.B. Gregory et al., Leading-order hadronic contributions to $g_{\mu}-2$, PoS (LATTICE 2013) 302 [arXiv:1311.4446] [INSPIRE].

[66] ALPHA collaboration, K. Jansen and R. Sommer, $O(\alpha)$ improvement of lattice $Q C D$ with two flavors of Wilson quarks, Nucl. Phys. B 530 (1998) 185 [Erratum ibid. B 643 (2002) 517] [hep-lat/9803017] [INSPIRE].

[67] M. Lüscher, Schwarz-preconditioned HMC algorithm for two-flavour lattice QCD, Comput. Phys. Commun. 165 (2005) 199 [hep-lat/0409106] [INSPIRE].

[68] M. Lüscher, Deflation acceleration of lattice QCD simulations, JHEP 12 (2007) 011 [arXiv:0710.5417] [INSPIRE]. 
[69] M. Marinkovic and S. Schaefer, Comparison of the mass preconditioned HMC and the DD-HMC algorithm for two-flavour QCD, PoS(LATTICE 2010) 031 [arXiv:1011.0911] [INSPIRE].

[70] P. Fritzsch et al., The strange quark mass and Lambda parameter of two flavor QCD, Nucl. Phys. B 865 (2012) 397 [arXiv: 1205.5380] [INSPIRE].

[71] P. Fritzsch, private communication, (2014).

[72] S. Lottini and R. Sommer, private communication, (2015).

[73] J. Heitger, G.M. von Hippel, S. Schaefer and F. Virotta, Charm quark mass and D-meson decay constants from two-flavour lattice QCD, PoS(LATTICE 2013) 475 [arXiv:1312.7693] [INSPIRE].

[74] M. Lüscher, S. Sint, R. Sommer and P. Weisz, Chiral symmetry and $O(a)$ improvement in lattice QCD, Nucl. Phys. B 478 (1996) 365 [hep-lat/9605038] [INSPIRE].

[75] M. Della Morte, R. Hoffmann, F. Knechtli, R. Sommer and U. Wolff, Non-perturbative renormalization of the axial current with dynamical Wilson fermions, JHEP 07 (2005) 007 [hep-lat/0505026] [INSPIRE].

[76] S. Sint and P. Weisz, Further results on $O(a)$ improved lattice QCD to one loop order of perturbation theory, Nucl. Phys. B 502 (1997) 251 [hep-lat/9704001] [INSPIRE].

[77] H. Horch, G. Herdoíza, B. Jäger, H. Wittig, M. Della Morte and A. Jüttner, Computing the Adler function from the vacuum polarization function, PoS (LATTICE 2013) 304 [arXiv: 1311.6975] [INSPIRE].

[78] C. Aubin, T. Blum, P. Chau, M. Golterman, S. Peris and C. Tu, Finite-volume effects in the muon anomalous magnetic moment on the lattice, Phys. Rev. D 93 (2016) 054508 [arXiv: 1512.07555] [INSPIRE].

[79] Budapest-Marseille-Wuppertal collaboration, R. Malak, Z. Fodor, C. Hölbling, L. Lellouch, A. Sastre and K. Szabo, Finite-volume corrections to the leading-order hadronic contribution to $g_{\mu}-2$, PoS (LATTICE2014) 161 [arXiv: 1502.02172] [INSPIRE].

[80] S. Güsken, U. Löw, K.H. Mütter, R. Sommer, A. Patel and K. Schilling, Nonsinglet Axial Vector Couplings of the Baryon Octet in Lattice QCD, Phys. Lett. B 227 (1989) 266 [INSPIRE].

[81] APE collaboration, M. Albanese et al., Glueball Masses and String Tension in Lattice QCD, Phys. Lett. B 192 (1987) 163 [InSPIRE].

[82] Particle Data Group collaboration, W.M. Yao et al., Review of Particle Physics, J. Phys. G 33 (2006) 1 [INSPIRE].

[83] S. Dürr et al., Ab-Initio Determination of Light Hadron Masses, Science 322 (2008) 1224 [arXiv: 0906.3599] [INSPIRE].

[84] A. Francis et al., The leading hadronic contribution to (g-2) of the muon: The chiral behavior using the mixed representation method, PoS (LATTICE2014) 127 [arXiv:1410.7491] [INSPIRE].

[85] M. Cè, L. Giusti and S. Schaefer, Domain decomposition, multi-level integration and exponential noise reduction in lattice QCD, Phys. Rev. D 93 (2016) 094507 [arXiv: 1601.04587 ] [INSPIRE].

[86] M. Cè, L. Giusti and S. Schaefer, A local factorization of the fermion determinant in lattice QCD, Phys. Rev. D 95 (2017) 034503 [arXiv: 1609. 02419] [InSPIRE]. 
[87] M. Lüscher, Signatures of unstable particles in finite volume, Nucl. Phys. B 364 (1991) 237 [INSPIRE].

[88] H.B. Meyer, Lattice QCD and the Timelike Pion Form Factor, Phys. Rev. Lett. 107 (2011) 072002 [arXiv: 1105.1892] [INSPIRE].

[89] X. Feng, S. Aoki, S. Hashimoto and T. Kaneko, Timelike pion form factor in lattice QCD, Phys. Rev. D 91 (2015) 054504 [arXiv: 1412.6319] [INSPIRE].

[90] J. Bulava, B. Hörz, B. Fahy, K.J. Juge, C. Morningstar and C.H. Wong, Pion-pion scattering and the timelike pion form factor from $N_{\mathrm{f}}=2+1$ lattice QCD simulations using the stochastic LapH method, PoS (LATTICE 2015) 069 [arXiv:1511.02351] [INSPIRE].

[91] F. Erben, J. Green, D. Mohler and H. Wittig, Towards extracting the timelike pion form factor on CLS 2-flavour ensembles, PoS (LATTICE2016) 382 [arXiv:1611.06805] [INSPIRE].

[92] G.J. Gounaris and J.J. Sakurai, Finite width corrections to the vector meson dominance prediction for $\rho \rightarrow e^{+} e^{-}$, Phys. Rev. Lett. 21 (1968) 244 [INSPIRE].

[93] M. Golterman, K. Maltman and S. Peris, Chiral extrapolation of the leading hadronic contribution to the muon anomalous magnetic moment, Phys. Rev. D 95 (2017) 074509 [arXiv: 1701.08685] [INSPIRE].

[94] J. Bijnens and J. Relefors, Chiral Perturbation Theory at Finite Volume and/or with Twisted Boundary Conditions, PoS (LATTICE2016) 282 [arXiv: 1611.06068] [INSPIRE].

[95] I.S. Gradshteyn and I.M. Ryshik, Table of Integrals, Series, and Products, 5th edition, Academic Press, London, U.K. (1994).

[96] Wolfram Research, Inc., Mathematica 9.0, (2012).

[97] C. Hanhart, A New Parameterization for the Pion Vector Form Factor, Phys. Lett. B 715 (2012) 170 [arXiv: 1203.6839] [InSPIRE].

[98] M. Benayoun et al., Hadronic contributions to the muon anomalous magnetic moment Workshop. $(g-2)_{\mu}$ : Quo vadis? Workshop. Mini proceedings, arXiv:1407.4021.

[99] M. Della Morte and A. Jüttner, Quark disconnected diagrams in chiral perturbation theory, JHEP 11 (2010) 154 [arXiv: 1009.3783] [INSPIRE].

[100] J. Bijnens and J. Relefors, Connected, Disconnected and Strange Quark Contributions to HVP, JHEP 11 (2016) 086 [arXiv:1609.01573] [INSPIRE].

[101] G.S. Bali, S. Collins and A. Schäfer, Effective noise reduction techniques for disconnected loops in Lattice QCD, Comput. Phys. Commun. 181 (2010) 1570 [arXiv:0910.3970] [INSPIRE].

[102] V. Gülpers, G. von Hippel and H. Wittig, Scalar pion form factor in two-flavor lattice QCD, Phys. Rev. D 89 (2014) 094503 [arXiv:1309.2104] [INSPIRE]. 